\title{
27. DATA REPORT: MINERALOGY AND Sr- AND Nd-ISOTOPIC COMPOSITION OF GABBROIC OCEANIC CRUST RECOVERED FROM HOLES 923A AND 921E IN THE MARK AREA ${ }^{1}$
}

\author{
Norie Fujibayashi, ${ }^{2}$ Hiroo Kagami, ${ }^{3}$ and Yoshiyuki Oishi $^{3}$
}

\begin{abstract}
Gabbroic rocks recovered from Holes 923A and 921E in the MARK area include troctolite, clinopyroxene-olivine gabbro, olivine-clinopyroxene gabbro, gabbro, gabbronorite, oxide gabbro, and quartz diorite. Clinopyroxene and plagioclase occur in all rock types, whereas olivine is supplanted by orthopyroxene in the evolved rock types and in the intercumulus stage. Forsterite (Fo) content of olivine, magnesium number (Mg\#) of pyroxenes, and anorthite (An) content of plagioclase systematically decrease with decreasing modal olivine content, and plagioclase is most sodic in the quartz diorite. Sr- and Nd-isotopic compositions are characteristic of N-type MORB.
\end{abstract}

\section{INTRODUCTION}

Gabbroic oceanic crust was drilled at the Mid-Atlantic Ridge near the Kane Fracture Zone (MARK, $23^{\circ} \mathrm{N}$ ) during Leg 153 . The rock types obtained span a wide range, including troctolite, olivine gabbro, gabbro, gabbronorite, oxide gabbro, and quartz diorite. Petrography, mineralogy, and $\mathrm{Sr}$ - and $\mathrm{Nd}$-isotopes are reported for a suite of 27 gabbroic rocks from Holes $923 \mathrm{~A}\left(23^{\circ} 32.55^{\prime} \mathrm{N}, 45^{\circ} 01.90^{\prime} \mathrm{W}\right)$ and $921 \mathrm{E}\left(23^{\circ} 32.40^{\prime} \mathrm{N}, 45^{\circ} 01.85^{\prime} \mathrm{W}\right)$ on the western median valley wall of the Mid-Atlantic Ridge about $10 \mathrm{~km}$ south of the Kane Transform Fault (Table 1).

\section{PETROGRAPHY}

Troctolite

Troctolites occur as thin (a few centimeters) layers or as dikes emplaced in gabbro, clinopyroxene-olivine gabbro, and olivine-clinopyroxene gabbro. Sample 153-923A-8R-2, Piece 1, is a thin dike. Sample 153-923A-12R-1, Piece 4B, is composed of layered troctolite and clinopyroxene-olivine gabbro; the troctolite part is labeled 153923A-12R-1, Piece 4B-Tr. These troctolites have large, tabular but anhedral, plagioclase, and anhedral olivine with less than $5 \%$ interstitial clinopyroxene and rare orthopyroxene. Orthopyroxene occurs partly rimming olivine grains.

\section{Clinopyroxene-Olivine Gabbro}

Clinopyroxene-olivine gabbro is a subtype of olivine gabbro after the IUGS classification scheme (Streckeisen, 1976), as is olivine-clinopyroxene gabbro, which is distinguished by a modal olivine/clinopyroxene ratio less than 1 .

Clinopyroxene-olivine gabbro is composed of olivine, clinopyroxene, plagioclase, and occasional small amounts of ilmenite and Ni-Fe sulfide. Samples 153-923A-13R-1, Piece 6, and 153-923A$7 \mathrm{R}-2$, Piece 1 , have large olivine and plagioclase crystals (average length $2-4 \mathrm{~mm}$, up to $6 \mathrm{~mm}$ ) and fine interstitial clinopyroxene.

'Karson. J.A., Cannat, M., Miller, D.J., and Elthon, D. (Eds.), 1997. Proc. ODP, Sci. Results, 153: College Station, TX (Ocean Drilling Program).

${ }^{2}$ Department of Geology, Faculty of Education, Niigata University, Niigata, 950-21, Japan. fujib@ed.niigata-u.ac.jp Japan.
Some olivine grains include partly subhedral to anhedral plagioclase laths.

Samples 153-923A-7R-1, Piece 2B, 7R-2, Piece 8, 8R-2, Piece 6, and $9 \mathrm{R}-2$, Piece $8 \mathrm{~A}$, contain oikocrystic olivine. In these rocks, large oikocrystic olivine (up to $6-10 \mathrm{~mm}$ across) encloses plagioclase laths. Plagioclase and clinopyroxene crystals tend to be smaller (average $1 \mathrm{~mm}$ ) than olivine grains, and clinopyroxene usually occurs interstitially. Some plagioclase grains are large, as much as $6 \mathrm{~mm}$ in length.

Sample 153-923A-12R-1, Piece 4B-COGb, is characterized by poikilitic clinopyroxene that encloses rounded olivine and plagioclase laths. The grain size of poikilitic clinopyroxene is as much as a few centimeters across.

\section{Olivine-Clinopyroxene Gabbro}

Most of the olivine-clinopyroxene gabbros are primarily equigranular, and are often modified into a porphyroclastic texture. Samples 153-923A-5R-1, Piece 2A, 9R-1, Piece 3D, 9R-2, Piece 9A, 10R-3, Piece 1, 153-921E-2R-1, Piece 6, and 4R-1, Piece 5, show primary equigranular texture, and are composed of plagioclase, clinopyroxene, olivine, and ilmenite. Samples 153-923A-2R-1, Piece 3, 9R3, Piece 3B (sheared), 10R-2, Piece 2A, 11R-1, Piece 12, and 11R-2, Piece 3, are somewhat sheared and show porphyroclastic textures.

An olivine-clinopyroxene gabbro, Sample 153-921E-7R-1, Piece $\mathrm{B}$, is characterized by poikilitic clinopyroxene. The poikilitic clinopyroxene is up to $2-3 \mathrm{~cm}$ across and encloses anhedral olivine and plagioclase crystals. Orthopyroxene rarely rims olivine grains.

\section{Gabbro}

Gabbro is composed of clinopyroxene, plagioclase, and minor amounts of olivine $(<5 \%)$, orthopyroxene $(<1 \%)$, and opaque minerals $(1 \%-2 \%)$. Opaque minerals are ilmenite and iron sulfide, and the total amount is high, up to a few percent. Some of these gabbros are called microgabbros because of their highly recrystallized, finegrained texture.

Samples 153-923A-5R-1, Piece 2A, and 5R-3, Piece 1, are typical coarse-grained gabbro without deformation texture. They are composed of equigranular, anhedral plagioclase, olivine, clinopyroxene, and interstitial orthopyroxene and opaque minerals. Modal olivine is less than $5 \%$.

Sample 153-923A-3R-2, Piece 1B, is highly sheared gabbro, having porphyroclasts of olivine, clinopyroxene, and plagioclase. 
Table 1. List of gabbroic rocks studied, Holes 921E and 923A.

\begin{tabular}{|c|c|c|c|c|c|c|}
\hline $\begin{array}{l}\text { Hole, core, } \\
\text { section }\end{array}$ & $\begin{array}{c}\text { Piece } \\
\text { no. }\end{array}$ & $\begin{array}{c}\text { Interval } \\
(\mathrm{cm})\end{array}$ & Rock name & Texture & Lithologic name & Unit \\
\hline \multicolumn{7}{|l|}{$153-923 \mathrm{~A}-$} \\
\hline $2 \mathrm{R}-1$ & 3 & $16-19$ & Microgabbro & Lineated & Variably deformed gabbro and olivine gabbro & 1 \\
\hline $3 R-2$ & 1B & $22-26$ & Gabbro & Gneissic & Variably deformed gabbro and olivine gabbro & 1 \\
\hline $5 \mathrm{R}-1$ & $2 \mathrm{~A}$ & $4-10$ & Gabbro & Equigranular & Variably deformed gabbro and olivine gabbro & 1 \\
\hline $5 \mathrm{R}-3$ & 1 & $19-29$ & Gabbro & Equigranular & Variably deformed gabbro and olivine gabbro & 1 \\
\hline $7 \mathrm{R}-1$ & 2B & $17-21$ & Cpx-ol gabbro & Ol oikocryst & Variably deformed gabbro and olivine gabbro & 1 \\
\hline $7 \mathrm{R}-2$ & 1 & $12-17$ & Cpx-ol gabbro & Ol oikocryst & Interlayered troctolite and olivine gabbro & 2 \\
\hline 7R-2 & 8 & $84-92$ & Cpx-ol gabbro & Ol oikocryst & Interlayered troctolite and olivine gabbro & 2 \\
\hline 8R-2 & 1 & $3-7$ & Troctolite & Equigranular & Interlayered troctolite and olivine gabbro & 2 \\
\hline 8R-2 & 6 & $61-67$ & Cpx-ol gabbro & Ol oikocryst & Interlayered troctolite and olivine gabbro & 2 \\
\hline $9 \mathrm{R}-1$ & 3D & $113-119$ & Ol-cpx gabbro & Equigranular & Interlayered troctolite and olivine gabbro & 2 \\
\hline $9 \mathrm{R}-2$ & $8 \mathrm{~A}$ & $65-70$ & Cpx-ol gabbro & Ol oikocryst & Interlayered troctolite and olivine gabbro & 2 \\
\hline $9 \mathrm{R}-2$ & $9 \mathrm{~A}$ & $109-114$ & Ol-cpx gabbro & Equigranular & Interlayered troctolite and olivine gabbro & 2 \\
\hline 9R-3 & 3B & $33-42$ & Ol-cpx gabbro & Sheared & Interlayered troctolite and olivine gabbro & 2 \\
\hline $10 \mathrm{R}-2$ & $2 \mathrm{~A}$ & $70-76$ & Ol-cpx gabbro & Sheared & Interlayered troctolite and olivine gabbro & 2 \\
\hline $10 \mathrm{R}-3$ & 1 & $7-13$ & Ol-cpx gabbro & Equigranular & Interlayered troctolite and olivine gabbro & 2 \\
\hline $11 \mathrm{R}-1$ & 12 & $119-123$ & Ol-cpx gabbro & Sheared & Interlayered troctolite and olivine gabbro & 2 \\
\hline $11 \mathrm{R}-2$ & 3 & $34-38$ & Ol-cpx gabbro & Sheared & Interlayered troctolite and olivine gabbro & 2 \\
\hline $12 \mathrm{R}-1$ & 4B & $38-43$ & Troctolite & Equigranular & Poikilitic olivine gabbro and troctolite & 3 \\
\hline $12 \mathrm{R}-1$ & 4B & $38-43$ & Cpx-ol gabbro & Poikilitic & Poikilitic olivine gabbro and troctolite & 3 \\
\hline $13 \mathrm{R}-1$ & 6 & $33-38$ & Cpx-ol gabbro & Ol oikocryst & Troctolite and poikilitic olivine gabbro & 4 \\
\hline \multicolumn{7}{|l|}{$153-921 \mathrm{E}-$} \\
\hline $2 \mathrm{R}-1$ & 6 & $77-84$ & Ol-cpx gabbro & Equigranular & Very coarse -grained to pegmatitic gabbro & 1 \\
\hline $3 \mathrm{R}-1$ & 5 & $37-41$ & Oxide gabbro & Sheared & Heterogeneous poikilitic olivine gabbro & 2 \\
\hline $4 \mathrm{R}-1$ & 5 & $39-44$ & Ol-cpx gabbro & Equigranular & Heterogeneous poikilitic olivine gabbro & 2 \\
\hline $7 \mathrm{R}-1$ & 4 & $25-30$ & Quartz diorite & Orthocumulate & Heterogeneous poikilitic olivine gabbro & 2 \\
\hline 7R-1 & 9B & $119-123$ & Öl-cpx gabbro & Poikilitic & Heterogeneous poikilitic olivine gabbro & 2 \\
\hline $8 \mathrm{R}-1$ & 1 & $0-6$ & Quartz diorite & Orthocumulate & Varitextured gabbro/olivine gabbro & 3 \\
\hline $8 \mathrm{R}-1$ & $7 \mathrm{~B}$ & $52-55$ & Gabbronorite & Sheared & Varitextured gabbro/olivine gabbro & 3 \\
\hline
\end{tabular}

Notes: $\mathrm{Cpx}=$ clinopyroxene $\mathrm{Ol}=$ olivine.

Microgabbro consists of a foliated fine-grained equigranular aggregate of clinopyroxene, plagoiclase, and brown hornblende, sometimes associated with olivine. Sample 153-923A-2R-1, Piece 3, is a typical microgabbro. Olivine and clinopyroxene occur rarely as porphyroclasts (1-2 $\mathrm{mm}$ across). Brown hornblende substitutes at the margins of clinopyroxene grains.

\section{Gabbronorite}

Gabbronorite generally occurs as a porphyroclastic dike intruded into the olivine gabbro. Gabbronorite (Sample 153-921E-8R-1, Piece 7B) was collected from such a dike, whose thickness is around 1 or 2 $\mathrm{m}$. The sample is composed of plagioclase, clinopyroxene, orthopyroxene, and opaque oxides. Pyroxenes show exsolution texture, and are elongated by shear stress.

\section{Oxide Gabbro}

Oxide gabbro usually forms thin veins in olivine gabbro. Sample 153-921E-3R-1, Piece 5, is a small vein (a few $\mathrm{cm}$ thick) intruded into the shear zone formed at the boundary between Units 1 (pegmatitic gabbro) and 2 (olivine gabbro). The anhedral plagioclase, clinopyroxene, and orthopyroxene grains are primarily cemented by ilmenite and titanomagnetite, and are deformed into a porphyroclastic texture.

\section{Quartz Diorite}

Only two discrete samples of quartz diorite were recovered during Leg 153, both from Hole 921E: Samples 153-921E-7R-1, Piece 4, and $8 \mathrm{R}-1$, Piece 1 . These specimens are composed of plagioclase, clinopyroxene, orthopyroxene, ilmenite, titanomagnetite, and quartz. Plagioclase grains are large and euhedral to subhedral in shape. Clinopyroxene, orthopyroxene, ilmenite, titanomagnetite, and quartz are the intergranular minerals. Pyroxenes are relict minerals and occur in the center part of the hornblendes and clay minerals. Clinopyroxene is surrounded by brown hornblende. Orthopyroxene is replaced by muscovite, chlorite, green hornblende, and so forth, at the margins.

\section{MINERALOGY}

Mineral compositions of plagioclase, olivine, and pyroxenes in gabbroic rocks from Holes $923 \mathrm{~A}$ and $921 \mathrm{E}$ were determined using the JEOL JXA-8600 Electron Microprobe at Niigata University. Standard silicate analyses were performed at an accelerating potential of $15 \mathrm{kV}$, a beam current of $1.3 \times 10^{-8} \mathrm{~A}$, and a spot size of $10 \mu \mathrm{m}$. The standards used for calibration were diopside $(\mathrm{Si}, \mathrm{Ca})$, synthetic $\mathrm{TiO}_{2}, \mathrm{Al}_{2} \mathrm{O}_{3}, \mathrm{Cr}_{2} \mathrm{O}_{3}, \mathrm{Fe}_{2} \mathrm{O}_{3}, \mathrm{NiO}$, periclase (Mg), jadeite (Na), and adularia $(\mathrm{K})$. Consistency of the data was checked by daily analyses of a kaersutite standard. Representative analyses are listed in Tables 2 , 3,4 , and 5 .

\section{Plagioclase}

Plagioclases in troctolite and clinopyroxene-olivine gabbro have high maximum anorthite (An) contents, typically $>72$ (Fig. 1, Table 2 ). Plagioclases in olivine-clinopyroxene gabbro are less calcic, but still have maximum An contents $>60$. The maximum An contents of plagioclases in gabbro are less than 60 . Porphyroclastic grains in gabbronorite have homogeneous An ranges between 45 and 47 . In oxide gabbro, some grains have calcic cores with An of 69-71, and the others have sodic cores with An of 45. Plagioclase grains in quartz diorite are sodic, having homogeneous cores characterized by An of 2627 , rimmed by a more sodic solution, whose An equals 7 .

\section{Olivine}

Olivine grains in troctolite and clinopyroxene-olivine gabbro have magnesian compositions with a forsterite (Fo) content $>76$, up to 86 (Fig. 2, Table 3). Olivines in Samples 153-923A-12R-1, Piece 4B-Tr, 12R-1, Piece 4B-COGb, 13R-1, Piece 6, and 7R-2, Piece 1, are magnesian, and have $\mathrm{NiO}$ contents greater than $0.24 \mathrm{wt} \%$ (Figs. $2,3)$. Maximum Fo contents of olivine in olivine-clinopyroxene gabbro generally range from 72 to 74 , except in Samples 153-921E-4R1, Piece 5, and 153-921E-7R-1, Piece 9B, which equal 75 and 78, respectively. Olivines in gabbro samples (Samples 153-923A-2R-1, Piece 3, and 5R-1, Piece 2A) have maximum Fo values of 73 and 74 , 
Table 2. Representative microprobe analyses of plagioclase for gabbroic rocks from Holes 921E and 923A.

\begin{tabular}{|c|c|c|c|c|c|c|c|c|c|c|c|c|c|}
\hline Hole: & $923 \mathrm{~A}$ & $923 \mathrm{~A}$ & $923 \mathrm{~A}$ & $923 \mathrm{~A}$ & $923 \mathrm{~A}$ & $923 \mathrm{~A}$ & $923 \mathrm{~A}$ & $923 \mathrm{~A}$ & $923 \mathrm{~A}$ & $923 \mathrm{~A}$ & $923 \mathrm{~A}$ & $923 \mathrm{~A}$ & $923 \mathrm{~A}$ \\
\hline Core, section: & $2 \mathrm{R}-3$ & $3 R-2$ & $3 R-2$ & $3 R-2$ & SR-1 & $5 R-1$ & $5 R-3$ & $5 R-3$ & $7 R-1$ & $7 R-1$ & $7 R-1$ & $7 \mathrm{R}-2$ & $7 \mathrm{R}-2$ \\
\hline Piece no.: & 1 & 1 & 1 & 1 & $2 \mathrm{~A}$ & $2 \mathrm{~A}$ & 1 & 1 & $2 \mathrm{~B}$ & $2 B$ & $2 \mathrm{~B}$ & 1 & 1 \\
\hline Rock name: & $\mathrm{Gb}$ & $\mathrm{Gb}$ & $\mathrm{Gb}$ & $\mathrm{Gb}$ & $\mathrm{Gb}$ & $\mathrm{Gb}$ & $\mathrm{Gb}$ & $\mathrm{Gb}$ & $\mathrm{COGb}$ & $\mathrm{COGb}$ & COGb & COGb & COGb \\
\hline $\mathrm{SiO}_{2}$ & 54.21 & 53.60 & 55.23 & 56.95 & 53.31 & 54.47 & 53.53 & 55.71 & 49.18 & 53.30 & 52.93 & 49.75 & 52.66 \\
\hline $\mathrm{TiO}_{2}$ & 0.00 & 0.05 & 0.04 & 0.01 & 0.04 & 0.04 & 0.07 & 0.13 & 0.07 & 0.06 & 0.05 & 0.02 & 0.02 \\
\hline $\mathrm{Al}_{2} \mathrm{O}_{3}$ & 28.38 & 29.12 & 28.33 & 27.15 & 29.70 & 28.90 & 29.06 & 27.77 & 31.69 & 29.14 & 29.60 & 31.24 & 29.90 \\
\hline $\mathrm{FeO}$ & 0.10 & 0.36 & 0.20 & 0.22 & 0.21 & 0.31 & 0.32 & 0.31 & 0.31 & 0.18 & 0.25 & 0.22 & 0.29 \\
\hline $\mathrm{MnO}$ & 0.00 & 0.10 & 0.03 & 0.11 & 0.05 & 0.00 & 0.00 & 0.00 & 0.02 & 0.05 & 0.05 & 0.10 & 0.09 \\
\hline $\mathrm{MgO}$ & 0.02 & 0.02 & 0.02 & 0.00 & 0.03 & 0.04 & 0.02 & 0.01 & 0.04 & 0.03 & 0.03 & 0.04 & 0.03 \\
\hline $\mathrm{CaO}$ & 11.09 & 11.63 & 10.35 & 9.21 & 11.89 & 10.95 & 11.54 & 10.04 & 14.93 & 11.54 & 12.23 & 14.63 & 12.76 \\
\hline $\mathrm{Na}_{2} \mathrm{O}$ & 5.04 & 4.72 & 5.75 & 6.28 & 4.69 & 5.00 & 4.71 & 5.68 & 2.88 & 4.74 & 4.41 & 3.13 & 4.27 \\
\hline $\mathrm{K}_{2} \mathrm{O}$ & 0.00 & 0.07 & 0.10 & 0.11 & 0.05 & 0.06 & 0.06 & 0.09 & 0.02 & 0.04 & 0.03 & 0.03 & 0.05 \\
\hline $\begin{array}{l}\text { Total } \\
\text { Atomic no. }\end{array}$ & $\begin{array}{c}98.83 \\
(\mathrm{O}=32)\end{array}$ & 99.68 & 100.04 & 100.03 & 99.97 & 99.76 & 99.31 & 99.74 & 99.12 & 99.08 & 99.59 & 99.15 & 100.06 \\
\hline $\mathrm{Si}$ & 9.886 & 9.731 & 9.954 & 10.226 & 9.651 & 9.846 & 9.7425 & 10.055 & 9.064 & 9.722 & 9.625 & 9.160 & 9.552 \\
\hline $\mathrm{Ti}$ & 0.000 & 0.007 & 0.005 & 0.002 & 0.006 & 0.005 & 0.010 & 0.017 & 0.009 & 0.008 & 0.007 & 0.003 & 0.003 \\
\hline $\mathrm{Al}$ & 6.100 & 6.231 & 6.017 & 5.746 & 6.337 & 6.156 & 6.234 & 5.907 & 6.885 & 6.265 & 6.343 & 6.778 & 6.391 \\
\hline $\mathrm{Fe}$ & 0.014 & 0.054 & 0.029 & 0.033 & 0.031 & 0.047 & 0.049 & 0.047 & 0.047 & 0.027 & 0.038 & 0.034 & 0.044 \\
\hline $\mathrm{Mn}$ & 0.000 & 0.016 & 0.005 & 0.016 & 0.007 & 0.001 & 0.000 & 0.000 & 0.002 & 0.008 & 0.008 & 0.015 & 0.014 \\
\hline $\mathrm{Mg}$ & 0.005 & 0.005 & 0.006 & 0.001 & 0.008 & 0.010 & 0.005 & 0.003 & 0.010 & 0.008 & 0.009 & 0.010 & 0.009 \\
\hline $\mathrm{Ca}$ & 2.167 & 2.263 & 2.001 & 1.772 & 2.308 & 2.122 & 2.252 & 1.942 & 2.949 & 2.257 & 2.384 & 2.887 & 2.481 \\
\hline $\mathrm{Na}$ & 1.781 & 1.663 & 2.009 & 2.186 & 1.647 & 1.753 & 1.661 & 1.989 & 1.029 & 1.675 & 1.555 & 1.117 & 1.500 \\
\hline $\mathrm{K}$ & 0.000 & 0.016 & 0.022 & 0.024 & 0.011 & 0.014 & 0.015 & 0.020 & 0.005 & 0.008 & 0.008 & 0.008 & 0.012 \\
\hline Total & 19.954 & 19.986 & 20.048 & 20.005 & 20.004 & 19.954 & 19.968 & 19.979 & 20.001 & 19.979 & 19.978 & 20.011 & 20.005 \\
\hline An & 54.9 & 57.4 & 49.6 & 44.5 & 58.2 & 54.6 & 57.3 & 49.2 & 74.0 & 57.3 & 60.4 & 72.0 & 62.1 \\
\hline $\mathrm{Ab}$ & 45.1 & 42.2 & 49.8 & 54.9 & 41.5 & 45.1 & 42.3 & 50.3 & 25.8 & 42.5 & 39.4 & 27.8 & 37.6 \\
\hline Or & 0.0 & 0.4 & 0.5 & 0.6 & 0.3 & 0.4 & 0.4 & 0.5 & 0.1 & 0.2 & 0.2 & 0.2 & 0.3 \\
\hline
\end{tabular}

Notes: $\operatorname{Tr}=$ troctolite, $\mathrm{COG} b=$ clinopyroxene-olivine gabbro, $\mathrm{OCG}=$ olivine-clinopyroxene gabbro, $\mathrm{Gb}=$ gabbro, $\mathrm{GbN}=$ gabbronorite, $\mathrm{OxGb}=\mathrm{oxide}$ gabbro, $\mathrm{Qz} \mathrm{Di}=\mathrm{quartz}$ diorite Oxide and elemental analyses are reported in weight percent; $\mathrm{An}, \mathrm{Ab}$, and $\mathrm{Or}$ are reported in mole percent.

Table 2 (continued).

\begin{tabular}{|c|c|c|c|c|c|c|c|c|c|c|c|c|c|}
\hline Hole: & $923 \mathrm{~A}$ & $923 \mathrm{~A}$ & $923 \mathrm{~A}$ & $923 \mathrm{~A}$ & $923 \mathrm{~A}$ & $923 \mathrm{~A}$ & $923 \mathrm{~A}$ & $923 \mathrm{~A}$ & $923 \mathrm{~A}$ & $923 \mathrm{~A}$ & $923 \mathrm{~A}$ & $923 \mathrm{~A}$ & $923 \mathrm{~A}$ \\
\hline Core, section: & $7 R-2$ & $7 \mathrm{R}-2$ & $7 R-2$ & $8 \mathrm{R}-2$ & $8 \mathrm{R}-2$ & $8 \mathrm{R}-2$ & $9 \mathrm{R}-1$ & $9 \mathrm{R}-1$ & 9R-2 & $9 \mathrm{R}-2$ & $9 R-2$ & 9R-2 & $9 \mathrm{R}-2$ \\
\hline Piece no.: & 8 & 8 & 8 & 1 & 1 & 6 & 3D & 3D & 8 & 8 & 8 & 9 & 9 \\
\hline Rock name: & $\mathrm{COGb}$ & $\mathrm{COGb}$ & COGb & $\operatorname{Tr}$ & $\operatorname{Tr}$ & COGb & OCGb & OCGb & $\mathrm{COGb}$ & COGb & $\mathrm{COGb}$ & OCGb & OCGb \\
\hline $\mathrm{SiO}_{2}$ & 49.84 & 51.53 & 52.52 & 50.37 & 53.46 & 48.71 & 52.50 & 51.52 & 49.45 & 51.23 & 53.36 & 53.62 & 51.47 \\
\hline $\mathrm{TiO}_{2}$ & 0.04 & 0.06 & 0.08 & 0.04 & 0.05 & 0.01 & 0.02 & 0.03 & 0.05 & 0.07 & 0.07 & 0.03 & 0.06 \\
\hline $\mathrm{Al}_{2} \mathrm{O}_{3}$ & 31.91 & 30.40 & 29.89 & 31.39 & 29.54 & 31.28 & 29.42 & 29.61 & 32.01 & 30.97 & 29.76 & 28.66 & 29.61 \\
\hline $\mathrm{FeO}$ & 0.39 & 0.38 & 0.34 & 0.37 & 0.25 & 0.24 & 0.28 & 0.30 & 0.21 & 0.24 & 0.24 & 0.18 & 0.32 \\
\hline $\mathrm{MnO}$ & 0.00 & 0.05 & 0.00 & 0.02 & 0.00 & 0.00 & 0.00 & 0.02 & 0.01 & 0.03 & 0.08 & 0.00 & 0.00 \\
\hline $\mathrm{MgO}$ & 0.05 & 0.02 & 0.04 & 0.06 & 0.04 & 0.03 & 0.00 & 0.01 & 0.04 & 0.02 & 0.04 & 0.02 & 0.06 \\
\hline $\mathrm{CaO}$ & 14.81 & 13.24 & 12.50 & 14.33 & 12.24 & 14.37 & 11.86 & 12.54 & 15.06 & 13.74 & 12.49 & 11.40 & 12.79 \\
\hline $\mathrm{Na}_{2} \mathrm{O}$ & 2.96 & 3.70 & 4.23 & 2.80 & 4.00 & 2.84 & 4.38 & 4.07 & 2.83 & 3.67 & 4.42 & 4.81 & 4.01 \\
\hline $\mathrm{K}_{2} \mathrm{O}$ & 0.04 & 0.05 & 0.07 & 0.03 & 0.03 & 0.03 & 0.04 & 0.03 & 0.01 & 0.00 & 0.04 & 0.00 & 0.00 \\
\hline $\begin{array}{l}\text { Total } \\
\text { Atomic no. }\end{array}$ & 100.05 & 99.43 & 99.67 & 99.41 & 99.60 & $\begin{array}{l}97.52 \\
(\mathrm{O}=32)\end{array}$ & 98.50 & 98.13 & 99.67 & 99.97 & 100.50 & 98.72 & 98.32 \\
\hline $\mathrm{Si}$ & 9.097 & 9.418 & 9.555 & 9.222 & 9.693 & 9.107 & 9.641 & 9.523 & 9.057 & 9.322 & 9.623 & 9.804 & 9.502 \\
\hline $\mathrm{Ti}$ & 0.006 & 0.008 & 0.011 & 0.006 & 0.007 & 0.001 & 0.002 & 0.003 & 0.007 & 0.009 & 0.010 & 0.004 & 0.008 \\
\hline $\mathrm{Al}$ & 6.863 & 6.548 & 6.409 & 6.772 & 6.311 & 6.892 & 6.367 & 6.451 & 6.911 & 6.641 & 6.324 & 6.176 & 6.442 \\
\hline $\mathrm{Fe}$ & 0.060 & 0.058 & 0.052 & 0.057 & 0.037 & 0.038 & 0.043 & 0.047 & 0.032 & 0.037 & 0.036 & 0.028 & 0.049 \\
\hline $\mathrm{Mn}$ & 0.000 & 0.008 & 0.000 & 0.003 & 0.000 & 0.000 & 0.000 & 0.003 & 0.001 & 0.005 & 0.011 & 0.000 & 0.000 \\
\hline $\mathrm{Mg}$ & 0.013 & 0.006 & 0.010 & 0.015 & 0.010 & 0.009 & 0.001 & 0.002 & 0.010 & 0.006 & 0.009 & 0.005 & 0.017 \\
\hline $\mathrm{Ca}$ & 2.899 & 2.594 & 2.438 & 2.812 & 2.379 & 2.880 & 2.334 & 2.486 & 2.957 & 2.680 & 2.415 & 2.235 & 2.532 \\
\hline $\mathrm{Na}$ & 1.049 & 1.311 & 1.493 & 0.993 & 1.405 & 1.030 & 1.559 & 1.457 & 1.005 & 1.294 & 1.546 & 1.705 & 1.435 \\
\hline $\mathrm{K}$ & 0.010 & 0.011 & 0.017 & 0.007 & 0.008 & 0.008 & 0.010 & 0.008 & 0.002 & 0.000 & 0.008 & 0.000 & 0.000 \\
\hline Total & 19.995 & 19.961 & 19.984 & 19.886 & 19.851 & 19.965 & 19.958 & 19.980 & 19.984 & 19.995 & 19.983 & 19.957 & 19.986 \\
\hline An & 73.3 & 66.2 & 61.8 & 73.8 & 62.7 & 73.5 & 59.8 & 62.9 & 74.6 & 67.4 & 60.8 & 56.7 & 63.8 \\
\hline $\mathrm{Ab}$ & 26.5 & 33.5 & 37.8 & 26.1 & 37.1 & 26.3 & 39.9 & 36.9 & 25.4 & 32.6 & 39.0 & 43.3 & 36.2 \\
\hline Or & 0.2 & 0.3 & 0.4 & 0.2 & 0.2 & 0.2 & $0.3^{3}$ & 0.2 & 0.1 & 0.0 & 0.2 & 0.0 & 0.0 \\
\hline
\end{tabular}

respectively, and those in Samples 153-923A-5R-3, Piece 1, and 3R2 , Piece 1 , have maximum Fo contents of 71 and 66 , respectively.

\section{Clinopyroxene}

Clinopyroxenes in troctolite, clinopyroxene-olivine gabbro, olivine-clinopyroxene gabbro, and gabbro have a significant range in diopside, endiopside, and augite contents (Fig. 4). Clinopyroxenes in primitive samples, such as troctolite (Samples 153-923A-12R-1, Piece 4B-Tr), and clinopyroxene-olivine gabbros (Samples 153923A-7R-2, Piece 1, 12R-1, Piece 4B-COGb, and 13R-1, Piece 6), plot entirely in the diopside and endiopside fields. The maximum magnesium numbers $(\mathrm{Mg} \#)$ of clinopyroxenes in these four samples are high (88 and 89 ) (Table 4). Clinopyroxenes in clinopyroxene-olivine gabbro (Samples 153-923A-7R-2, Piece 1, and 153-923A-12R1, Piece 4B-COGb) also have high $\mathrm{Cr}_{2} \mathrm{O}_{3}$ contents, up to 1.3 (wt\%) (Fig. 5). Clinopyroxenes in gabbronorite, oxide gabbro, and quartz diorite have low maximum $\mathrm{Mg} \#$ values, ranging from 64 to 74 , and plot along the boundary between the salite and augite fields (Fig. 4).

\section{Orthopyroxene}

Orthopyroxenes in troctolite, clinopyroxene-olivine gabbro, and olivine-clinopyroxene gabbro are classified as bronzite (Fig. 4). 
Table 2 (continued).

\begin{tabular}{|c|c|c|c|c|c|c|c|c|c|c|c|c|c|}
\hline Hole: & $923 \mathrm{~A}$ & $923 \mathrm{~A}$ & $923 \mathrm{~A}$ & $923 \mathrm{~A}$ & $923 \mathrm{~A}$ & $923 \mathrm{~A}$ & $923 \mathrm{~A}$ & $923 \mathrm{~A}$ & $923 \mathrm{~A}$ & $923 \mathrm{~A}$ & $923 \mathrm{~A}$ & $923 \mathrm{~A}$ & $923 \mathrm{~A}$ \\
\hline Core, section: & $9 R-3$ & $9 \mathrm{R}-3$ & $10 \mathrm{R}-2$ & $10 \mathrm{R}-2$ & $10 \mathrm{R}-3$ & $10 \mathrm{R}-3$ & $11 \mathrm{R}-1$ & $11 \mathrm{R}-1$ & $11 \mathrm{R}-2$ & $11 \mathrm{R}-2$ & $12 \mathrm{R}-1$ & $12 \mathrm{R}-1$ & $12 \mathrm{R}-1$ \\
\hline Piece no.: & 3 & $3 B$ & 2 & 2 & 1 & 1 & 12 & 12 & 3 & 3 & $4 B$ & $4 B$ & $4 B$ \\
\hline Rock name: & OCGb & OCGb & OCGb & OCGb & OCGb & OCGb & OCGb & OCGb & OCGb & OCGb & $\mathrm{Tr}$ & $\operatorname{Tr}$ & $\operatorname{Tr}$ \\
\hline $\begin{array}{l}\mathrm{SiO}_{2} \\
\mathrm{TiO}_{2} \\
\mathrm{Al}_{2} \mathrm{O}_{3} \\
\mathrm{FeO} \\
\mathrm{MnO} \\
\mathrm{MgO} \\
\mathrm{CaO} \\
\mathrm{Na}_{2} \mathrm{O} \\
\mathrm{K}_{2} \mathrm{O} \\
\text { Total } \\
\text { Atomic no. }\end{array}$ & $\begin{array}{l}53.03 \\
0.09 \\
29.46 \\
0.34 \\
0.02 \\
0.04 \\
12.43 \\
4.25 \\
0.04 \\
99.68\end{array}$ & $\begin{array}{l}54.24 \\
0.02 \\
29.03 \\
0.25 \\
0.00 \\
0.03 \\
11.67 \\
4.80 \\
0.05 \\
100.09\end{array}$ & $\begin{array}{l}52.75 \\
0.05 \\
29.78 \\
0.33 \\
0.06 \\
0.03 \\
12.49 \\
4.40 \\
0.03 \\
99.92\end{array}$ & $\begin{array}{l}54.86 \\
0.03 \\
28.59 \\
0.26 \\
0.08 \\
0.03 \\
10.79 \\
5.38 \\
0.05 \\
100.06\end{array}$ & $\begin{array}{l}52.61 \\
0.06 \\
29.79 \\
0.35 \\
0.02 \\
0.13 \\
12.43 \\
4.31 \\
0.07 \\
99.77\end{array}$ & $\begin{array}{l}54.04 \\
0.04 \\
28.79 \\
0.35 \\
0.01 \\
0.03 \\
11.37 \\
5.03 \\
0.05 \\
99.70\end{array}$ & $\begin{array}{l}52.84 \\
0.07 \\
29.45 \\
0.28 \\
0.00 \\
0.03 \\
12.58 \\
4.32 \\
0.04 \\
99.59\end{array}$ & $\begin{array}{l}54.23 \\
0.07 \\
28.23 \\
0.35 \\
0.00 \\
0.02 \\
11.11 \\
5.03 \\
0.06 \\
99.09\end{array}$ & $\begin{array}{l}53.13 \\
0.01 \\
28.81 \\
0.30 \\
0.03 \\
0.02 \\
11.72 \\
4.51 \\
0.06 \\
98.57\end{array}$ & $\begin{array}{l}54.50 \\
0.05 \\
28.16 \\
0.34 \\
0.04 \\
0.04 \\
11.01 \\
4.81 \\
0.05 \\
98.98\end{array}$ & $\begin{array}{r}48.78 \\
0.00 \\
32.27 \\
0.25 \\
0.01 \\
0.01 \\
15.56 \\
2.58 \\
0.00 \\
99.47 \\
(\mathrm{O}=32)\end{array}$ & $\begin{array}{r}50.49 \\
0.07 \\
30.92 \\
0.27 \\
0.00 \\
0.03 \\
14.29 \\
3.29 \\
0.00 \\
99.37\end{array}$ & $\begin{array}{r}51.01 \\
0.08 \\
30.40 \\
0.31 \\
0.03 \\
0.01 \\
13.54 \\
3.75 \\
0.00 \\
99.13\end{array}$ \\
\hline $\begin{array}{l}\mathrm{Si} \\
\mathrm{Ti} \\
\mathrm{Al} \\
\mathrm{Fe} \\
\mathrm{Mn} \\
\mathrm{Mg} \\
\mathrm{Ca} \\
\mathrm{Na} \\
\mathrm{K} \\
\text { Total }\end{array}$ & $\begin{array}{l}9.637 \\
0.012 \\
6.310 \\
0.052 \\
0.002 \\
0.011 \\
2.421 \\
1.497 \\
0.009 \\
19.950\end{array}$ & $\begin{array}{l}9.790 \\
0.003 \\
6.176 \\
0.037 \\
0.000 \\
0.009 \\
2.259 \\
1.679 \\
0.011 \\
19.964\end{array}$ & $\begin{array}{l}9.577 \\
0.007 \\
6.372 \\
0.050 \\
0.010 \\
0.007 \\
2.430 \\
1.548 \\
0.007 \\
20.008\end{array}$ & $\begin{array}{l}9.894 \\
0.004 \\
6.077 \\
0.040 \\
0.011 \\
0.008 \\
2.085 \\
1.880 \\
0.011 \\
20.009\end{array}$ & $\begin{array}{l}9.565 \\
0.008 \\
6.383 \\
0.053 \\
0.003 \\
0.034 \\
2.422 \\
1.519 \\
0.016 \\
20.003\end{array}$ & $\begin{array}{l}9.799 \\
0.005 \\
6.152 \\
0.053 \\
0.001 \\
0.009 \\
2.210 \\
1.768 \\
0.012 \\
20.009\end{array}$ & $\begin{array}{l}9.618 \\
0.009 \\
6.317 \\
0.043 \\
0.000 \\
0.007 \\
2.455 \\
1.523 \\
0.010 \\
19.981\end{array}$ & $\begin{array}{l}9.882 \\
0.009 \\
6.063 \\
0.054 \\
0.000 \\
0.006 \\
2.170 \\
1.776 \\
0.013 \\
19.972\end{array}$ & $\begin{array}{l}9.744 \\
0.001 \\
6.227 \\
0.046 \\
0.004 \\
0.006 \\
2.305 \\
1.603 \\
0.014 \\
19.950\end{array}$ & $\begin{array}{l}9.925 \\
0.006 \\
6.043 \\
0.051 \\
0.006 \\
0.010 \\
2.150 \\
1.699 \\
0.012 \\
19.903\end{array}$ & $\begin{array}{r}8.970 \\
0.000 \\
6.992 \\
0.038 \\
0.002 \\
0.003 \\
3.068 \\
0.921 \\
0.000 \\
19.994\end{array}$ & $\begin{array}{r}9.257 \\
0.010 \\
6.680 \\
0.042 \\
0.000 \\
0.009 \\
2.809 \\
1.171 \\
0.000 \\
19.978\end{array}$ & $\begin{array}{r}9.363 \\
0.011 \\
6.576 \\
0.047 \\
0.005 \\
0.004 \\
2.664 \\
1.335 \\
0.000 \\
20.005\end{array}$ \\
\hline $\begin{array}{l}\mathrm{An} \\
\mathrm{Ab} \\
\mathrm{Or}\end{array}$ & $\begin{array}{l}61.7 \\
38.1 \\
0.2\end{array}$ & $\begin{array}{l}57.2 \\
42.5 \\
0.3\end{array}$ & $\begin{array}{l}61.0 \\
38.8 \\
0.2\end{array}$ & $\begin{array}{l}52.4 \\
47.3 \\
0.3\end{array}$ & $\begin{array}{l}61.2 \\
38.4 \\
0.4\end{array}$ & $\begin{array}{l}55.4 \\
44.3 \\
0.3\end{array}$ & $\begin{array}{l}61.6 \\
38.2 \\
0.2\end{array}$ & $\begin{array}{l}54.8 \\
44.9 \\
0.3\end{array}$ & $\begin{array}{l}58.8 \\
40.9 \\
0.3\end{array}$ & $\begin{array}{l}55.7 \\
44.0 \\
0.3\end{array}$ & $\begin{array}{r}76.9 \\
23.1 \\
0.0\end{array}$ & $\begin{array}{r}70.6 \\
29.4 \\
0.0\end{array}$ & $\begin{array}{r}66.6 \\
33.4 \\
0.0\end{array}$ \\
\hline
\end{tabular}

Table 2 (continued).

\begin{tabular}{|c|c|c|c|c|c|c|c|c|c|c|c|c|c|}
\hline Hole: & $923 \mathrm{~A}$ & $923 \mathrm{~A}$ & $923 \mathrm{~A}$ & $923 \mathrm{~A}$ & $923 \mathrm{~A}$ & $923 \mathrm{~A}$ & $921 \mathrm{E}$ & $921 \mathrm{E}$ & $921 \mathrm{E}$ & $921 \mathrm{E}$ & $921 \mathrm{E}$ & $921 \mathrm{E}$ & $921 \mathrm{E}$ \\
\hline Core, section: & $12 R-1$ & $12 \mathrm{R}-1$ & $12 \mathrm{R}-1$ & 13R-1 & $13 \mathrm{R}-1$ & 13R-1 & $2 \mathrm{R}-1$ & $2 R-1$ & $2 \mathrm{R}-1$ & $3 R-1$ & $3 R-1$ & $3 R-1$ & $4 \mathrm{R}-1$ \\
\hline Piece no.: & $4 B$ & 4B & 4B & 6 & 6 & 6 & 6 & 6 & 6 & 5 & 5 & 5 & 5 \\
\hline Rock name: & COGb & COGb & COGb & COGb & COGb & $\mathrm{COGb}$ & OCGb & OCGb & OCGb & OxGb & $\mathrm{OxGb}$ & $\mathrm{OxGb}$ & OCGb \\
\hline $\mathrm{SiO}_{2}$ & 48.95 & 50.11 & 53.05 & 49.97 & 51.21 & 52.34 & 53.10 & 53.78 & 54.95 & 53.30 & 57.93 & 59.66 & 51.38 \\
\hline $\mathrm{TiO}_{2}$ & 0.00 & 0.05 & 0.03 & 0.04 & 0.06 & 0.06 & 0.01 & 0.01 & 0.01 & 0.03 & 0.00 & 0.00 & 0.03 \\
\hline $\mathrm{Al}_{2} \mathrm{O}_{3}$ & 32.26 & 31.48 & 30.01 & 31.88 & 30.95 & 30.24 & 29.38 & 28.90 & 28.20 & 30.91 & 28.04 & 26.25 & 29.89 \\
\hline $\mathrm{FeO}$ & 0.20 & 0.14 & 0.18 & 0.29 & 0.14 & 0.23 & 0.29 & 0.17 & 0.22 & 0.23 & 0.13 & 0.20 & 0.36 \\
\hline $\mathrm{MnO}$ & 0.05 & 0.07 & 0.05 & 0.00 & 0.00 & 0.00 & 0.00 & 0.01 & 0.01 & 0.00 & 0.00 & 0.00 & 0.00 \\
\hline $\mathrm{MgO}$ & 0.13 & 0.04 & 0.11 & 0.03 & 0.03 & 0.02 & 0.04 & 0.04 & 0.03 & 0.03 & 0.01 & 0.03 & 0.06 \\
\hline $\mathrm{CaO}$ & 15.70 & 14.45 & 12.84 & 14.97 & 13.85 & 13.13 & 12.15 & 11.39 & 10.39 & 13.39 & 9.84 & 8.25 & 13.17 \\
\hline $\mathrm{Na}_{2} \mathrm{O}$ & 2.36 & 2.97 & 4.00 & 3.01 & 3.66 & 4.11 & 4.83 & 5.19 & 5.82 & 3.08 & 4.82 & 5.45 & 4.21 \\
\hline $\mathrm{K}_{2} \mathrm{O}$ & 0.03 & 0.04 & 0.04 & 0.01 & 0.02 & 0.02 & 0.05 & 0.08 & 0.07 & 0.01 & 0.07 & 0.06 & 0.04 \\
\hline $\begin{array}{l}\text { Total } \\
\text { Atomic no. }\end{array}$ & 99.69 & 99.33 & 100.32 & 100.20 & 99.90 & $\begin{array}{l}100.15 \\
(O=32)\end{array}$ & 99.85 & 99.56 & 99.69 & 100.96 & 100.84 & 99.89 & 99.14 \\
\hline Si & 8.978 & 9.186 & 9.579 & 9.106 & 9.323 & 9.488 & 9.645 & 9.769 & 9.944 & 9.536 & 10.253 & 10.601 & 9.434 \\
\hline $\mathrm{Ti}_{1}$ & 0.000 & 0.007 & 0.004 & 0.005 & 0.008 & 0.007 & 0.001 & 0.002 & 0.001 & 0.005 & 0.000 & 0.000 & 0.005 \\
\hline Al & 6.973 & 6.800 & 6.385 & 6.846 & 6.640 & 6.460 & 6.288 & 6.188 & 6.015 & 6.517 & 5.848 & 5.497 & 6.470 \\
\hline $\mathrm{Fe}$ & 0.031 & 0.021 & 0.028 & 0.045 & 0.021 & 0.035 & 0.045 & 0.026 & 0.034 & 0.034 & 0.019 & 0.030 & 0.055 \\
\hline $\mathrm{Mn}$ & 0.007 & 0.010 & 0.008 & 0.000 & 0.000 & 0.000 & 0.000 & 0.001 & 0.001 & 0.000 & 0.000 & 0.000 & 0.000 \\
\hline $\mathrm{Mg}$ & 0.035 & 0.011 & 0.031 & 0.007 & 0.007 & 0.006 & 0.009 & 0.010 & 0.009 & 0.007 & 0.002 & 0.007 & 0.016 \\
\hline $\mathrm{Ca}$ & 3.088 & 2.840 & 2.485 & 2.925 & 2.703 & 2.551 & 2.366 & 2.217 & 2.015 & 2.568 & 1.868 & 1.571 & 2.593 \\
\hline $\mathrm{Na}$ & 0.841 & 1.055 & 1.400 & 1.064 & 1.290 & 1.445 & 1.701 & 1.828 & 2.043 & 1.067 & 1.652 & 1.876 & 1.498 \\
\hline $\mathrm{K}$ & 0.007 & 0.008 & 0.009 & 0.003 & 0.004 & 0.006 & 0.012 & 0.019 & 0.016 & 0.001 & 0.015 & 0.013 & 0.009 \\
\hline Total & 19.959 & 19.939 & 19.928 & 20.000 & 19.997 & 19.999 & 20.067 & 20.058 & 20.077 & 19.735 & 19.657 & 19.595 & 20.080 \\
\hline An & 78.5 & 72.8 & 63.8 & 73.3 & 67.6 & 63.8 & 58.0 & 54.6 & 49.5 & 70.6 & 52.8 & 45.4 & 63.2 \\
\hline $\mathrm{Ab}$ & 21.4 & 27.0 & 36.0 & 26.7 & 32.3 & 36.1 & 41.7 & 45.0 & 50.1 & 29.3 & 46.7 & 54.2 & 36.5 \\
\hline Or & 0.2 & 0.2 & 0.2 & 0.1 & 0.1 & 0.1 & 0.3 & 0.5 & 0.4 & 0.0 & 0.4 & 0.4 & 0.2 \\
\hline
\end{tabular}

Among those, crystals in Samples 153-923A-12R-1, Piece 4B-COGb and - $\mathrm{Tr}$, have the highest $\mathrm{Mg} \#$ (76-77). Orthopyroxenes in most gabbro samples also plot in the bronzite field, whereas those in gabbronorite plot near the boundary between bronzite and hypersthene. Orthopyroxenes in oxide gabbro and quartz diorite are classified as hypersthene. Neither pigeonite nor inverted pigeonite occur in the examined samples.

\section{Sr- AND Nd-ISOTOPIC COMPOSITION}

Six samples of various rock types were selected for isotopic analyses. Samples were crushed in a tungsten carbide mill to a few milli- meters in size, and then warmed on a hot plate and washed ultrasonically in distilled water until no $\mathrm{AgCl}$ was produced by reaction with $2 \% \mathrm{AgNO}_{3}$. The sample for bulk analysis was segregated at this stage and ground in a tungsten carbide mill. The washed chips were again crushed for mineral separates to the 60 mesh size, and then warmed in $6-\mathrm{N} \mathrm{HCl}$ for $30 \mathrm{~min}$ to obtain fresh mineral separates by dissolving clay minerals. Samples then were cleaned ultrasonically and rinsed in distilled water. Plagioclase and pyroxene were separated using isodynamic magnetic separators.

Sr- and Nd-isotope analyses were performed using a Finnigan MAT 261 multicollector mass spectrometer at the Institute of Study for Earth's Interior, Okayama University. The Sr and Nd extractions followed the procedures described by Kagami et al. $(1987,1989)$. 
Table 2 (continued).

\begin{tabular}{|c|c|c|c|c|c|c|c|c|c|c|c|}
\hline Hole: & $921 \mathrm{E}$ & $921 \mathrm{E}$ & $921 \mathrm{E}$ & $921 \mathrm{E}$ & $921 \mathrm{E}$ & $921 \mathrm{E}$ & $921 \mathrm{E}$ & $921 \mathrm{E}$ & $921 \mathrm{E}$ & $921 \mathrm{E}$ & 921E \\
\hline Core, section: & $4 \mathrm{R}-1$ & $7 R-1$ & $7 \mathrm{R}-1$ & $7 \mathrm{R}-1$ & $7 R-1$ & $7 R-1$ & $8 \mathrm{R}-1$ & $8 \mathrm{R}-1$ & $8 R-1$ & $8 \mathrm{R}-1$ & $8 R-1$ \\
\hline Piece no.: & 5 & 4 & 4 & 4 & $9 \mathrm{~B}$ & $9 \mathrm{~B}$ & 1 & 1 & 1 & $7 \mathrm{~B}$ & $7 \mathrm{~B}$ \\
\hline Rock name: & OCGb & QzDi & QzDi & QzDi & OCGb & OCGb & QzDi & QzDi & QzDi & $\mathrm{GbN}$ & $\mathrm{GbN}$ \\
\hline $\mathrm{SiO}_{2}$ & 54.49 & 61.49 & 63.48 & 65.97 & 52.06 & 54.25 & 60.78 & 64.63 & 67.23 & 55.51 & 56.69 \\
\hline $\mathrm{TiO}_{2}$ & 0.06 & 0.00 & 0.00 & 0.00 & 0.00 & 0.00 & 0.00 & 0.00 & 0.00 & 0.11 & 0.02 \\
\hline $\mathrm{Al}_{2} \mathrm{O}_{3}$ & 27.93 & 23.68 & 22.90 & 21.15 & 30.80 & 29.48 & 23.90 & 21.50 & 20.56 & 27.11 & 27.22 \\
\hline $\mathrm{FeO}$ & 0.28 & 0.28 & 0.20 & 0.07 & 0.17 & 0.10 & 0.24 & 0.12 & 0.03 & 1.32 & 0.22 \\
\hline $\mathrm{MnO}$ & 0.03 & 0.00 & 0.00 & 0.00 & 0.02 & 0.02 & 0.00 & 0.00 & 0.00 & 0.01 & 0.00 \\
\hline $\mathrm{MgO}$ & 0.03 & 0.02 & 0.01 & 0.00 & 0.00 & 0.00 & 0.02 & 0.00 & 0.00 & 0.03 & 0.02 \\
\hline $\mathrm{CaO}$ & 11.15 & 5.80 & 4.59 & 2.43 & 13.52 & 12.00 & 5.83 & 3.17 & 1.66 & 9.75 & 9.46 \\
\hline $\mathrm{Na}_{2} \mathrm{O}$ & 5.54 & 8.71 & 9.28 & 10.94 & 3.75 & 4.96 & 8.82 & 10.03 & 11.27 & 6.11 & 6.24 \\
\hline $\mathrm{K}_{2} \mathrm{O}$ & 0.04 & 0.17 & 0.43 & 0.27 & 0.02 & 0.03 & 0.16 & 0.33 & 0.37 & 0.07 & 0.06 \\
\hline $\begin{array}{l}\text { Total } \\
\text { Atomic no. }\end{array}$ & 99.56 & 100.15 & $\begin{array}{l}100.88 \\
(\mathrm{O}=32)\end{array}$ & 100.83 & 100.36 & 100.84 & 99.74 & 99.79 & 101.11 & 100.01 & 99.92 \\
\hline $\mathrm{Si}$ & 9.901 & 10.939 & 11.173 & 11.550 & 9.418 & 9.731 & 10.870 & 11.445 & 11.707 & 10.053 & 10.194 \\
\hline $\mathrm{Ti}$ & 0.009 & 0.000 & 0.000 & 0.000 & 0.000 & 0.000 & 0.000 & 0.000 & 0.000 & 0.014 & 0.002 \\
\hline $\mathrm{Al}$ & 5.982 & 4.964 & 4.751 & 4.365 & 6.567 & 6.232 & 5.037 & 4.487 & 4.219 & 5.788 & 5.769 \\
\hline $\mathrm{Fe}$ & 0.043 & 0.042 & 0.029 & 0.010 & 0.026 & 0.016 & 0.036 & 0.017 & 0.005 & 0.200 & 0.032 \\
\hline Mn & 0.004 & 0.000 & 0.000 & 0.000 & 0.003 & 0.003 & 0.000 & 0.000 & 0.000 & 0.001 & 0.000 \\
\hline $\mathrm{Mg}$ & 0.007 & 0.005 & 0.003 & 0.001 & 0.001 & 0.000 & 0.005 & 0.000 & 0.000 & 0.009 & 0.006 \\
\hline $\mathrm{Ca}$ & 2.173 & 1.107 & 0.865 & 0.457 & 2.623 & 2.307 & 1.117 & 0.603 & 0.310 & 1.893 & 1.823 \\
\hline $\mathrm{Na}$ & 1.951 & 3.005 & 3.166 & 3.713 & 1.316 & 1.724 & 3.057 & 3.443 & 3.804 & 2.144 & 2.174 \\
\hline $\mathrm{K}$ & 0.010 & 0.038 & 0.096 & 0.061 & 0.005 & 0.007 & 0.036 & 0.074 & 0.081 & 0.016 & 0.014 \\
\hline Total & 20.080 & 20.100 & 20.083 & 20.155 & 19.959 & 20.019 & 20.159 & 20.070 & 20.126 & 20.119 & 20.014 \\
\hline An & 52.6 & 26.7 & 21.0 & 10.8 & 66.5 & 57.1 & 26.5 & 14.6 & 7.4 & 46.7 & 45.4 \\
\hline $\mathrm{Ab}$ & 47.2 & 72.4 & 76.7 & 87.8 & 33.4 & 42.7 & 72.6 & 83.6 & 90.7 & 52.9 & 54.2 \\
\hline Or & 0.2 & 0.9 & 2.3 & 1.4 & 0.1 & 0.2 & 0.9 & 1.8 & 1.9 & 0.4 & 0.4 \\
\hline
\end{tabular}

The extracted elements were loaded on Ta-filament in a double filament mode. Blanks for both $\mathrm{Sr}$ and Nd were less than $1 \mathrm{ng}$. The average ${ }^{87} \mathrm{Sr} /{ }^{86} \mathrm{Sr}$ ratio for the NBS987 standard was $0.710258 \pm 14( \pm$ represents an analytical reproducibility in terms of $2 \sigma$ ) and the average ${ }^{143} \mathrm{Nd} /{ }^{144} \mathrm{Nd}$ for the BCR-1 was $0.512612 \pm 11 .{ }^{87} \mathrm{Sr} /{ }^{86} \mathrm{Sr}$ was normalized to ${ }^{86} \mathrm{Sr} /{ }^{88} \mathrm{Sr}=0.1194 ;{ }^{143} \mathrm{Nd} /{ }^{144} \mathrm{Nd}$ was normalized to a value of ${ }^{146} \mathrm{Nd} /{ }^{144} \mathrm{Nd}=0.7219$. The results are listed in Table 6 . The ${ }^{143} \mathrm{Nd} /$ ${ }^{144} \mathrm{Nd}$ ratios are reported relative to ${ }^{143} \mathrm{Nd} /{ }^{144} \mathrm{Nd}=0.512640$ for $\mathrm{BCR}$ 1 (Wasserburg et al., 1981).

The ${ }^{87} \mathrm{Sr} /{ }^{86} \mathrm{Sr}$ ratio ranges from 0.70232 to 0.70260 , and ${ }^{143} \mathrm{Nd} /$ ${ }^{144} \mathrm{Nd}$ ranges from 0.513243 to 0.513252 . All the ${ }^{87} \mathrm{Sr} /{ }^{86} \mathrm{Sr}$ of analyzed samples are less than 0.7030 and in the range of N-type MORB (Wilson, 1989).

\section{ACKNOWLEDGMENTS}

We sincerely thank the scientists, Ocean Drilling Program staff, and crew of the JOIDES Resolution for their support during and after Leg 153. We also thank Dr. S. Miyashita, Dr. T. Shimura, and Mr. U. Sakai of Niigata University for their assistance with EPMA analyses.

\section{REFERENCES}

Kagami, H., Iwata, M., Sano, S., and Honma, H., 1987. Sr and Nd isotopic compositions and $\mathrm{Rb}, \mathrm{Sr}, \mathrm{Sm}$ and $\mathrm{Nd}$ concentrations of standard samples. Tech. Repts. ISEI, Okayama Univ., Ser. B, 4:1-16.

Kagami, H., Yokose, H., and Honma, H., 1989. ${ }^{87} \mathrm{Sr} /{ }^{86} \mathrm{Sr}$ and ${ }^{143} \mathrm{Nd} /{ }^{144} \mathrm{Nd}$ ratios of GSJ rock reference samples: JB-1a, JA-1 and JG-1a. Geochem. J., 23: 209-214.

Streckeisen, A., 1976. To each plutonic rock its proper name. Earth Sci. Rev, 12:1-33.

Wasserburg, G.J., Jacobsen, S.B., De Paolo, D.J., McCulloch, M.T., and Wen, T., 1981. Precise determination of $\mathrm{Sm} / \mathrm{Nd}$ ratios, $\mathrm{Sm}$ and Nd isotopic abundances in standard solutions. Geochim. Cosmochim. Acta, 45:2311-2323.

Wilson, M., 1989. Igneous Petrogenesis: A Global Tectonic Approach: London (Unwin Hyman).

Date of initial receipt: 6 September 1995

Date of acceptance: 1 February 1996

Ms 153SR-028 
Table 3. Representative microprobe analyses of olivine for gabbroic rocks from Holes 921E and 923A.

\begin{tabular}{|c|c|c|c|c|c|c|c|c|c|c|c|c|c|}
\hline Hole: & $923 \mathrm{~A}$ & $923 \mathrm{~A}$ & $923 \mathrm{~A}$ & $923 \mathrm{~A}$ & $923 \mathrm{~A}$ & $923 \mathrm{~A}$ & $923 \mathrm{~A}$ & $923 \mathrm{~A}$ & $923 \mathrm{~A}$ & $923 \mathrm{~A}$ & $923 \mathrm{~A}$ & $923 \mathrm{~A}$ & $923 \mathrm{~A}$ \\
\hline Core, section: & 2R-1 & 2R-1 & $3 \mathrm{R}-2$ & $3 R-2$ & $5 R-1$ & $5 R-3$ & $5 R-3$ & $7 \mathrm{R}-1$ & $7 R-1$ & $7 R-1$ & $7 R-2$ & 7R-2 & $7 \mathrm{R}-2$ \\
\hline Piece no.: & 3 & 3 & 1 & 1 & $2 \mathrm{~A}$ & 1 & 1 & $2 B$ & $2 \mathrm{~B}$ & $2 B$ & 1 & 1 & 8 \\
\hline Rock name: & $\mathrm{Gb}$ & $\mathrm{Gb}$ & $\mathrm{Gb}$ & $\mathrm{Gb}$ & $\mathrm{Gb}$ & $\mathrm{Gb}$ & $\mathrm{Gb}$ & COGb & COGb & COGb & COGb & COGb & $\mathrm{COGb}$ \\
\hline $\mathrm{SiO}_{2}$ & 37.94 & 37.65 & 37.05 & 36.68 & 38.44 & 37.70 & 37.68 & 38.63 & 38.13 & 37.23 & 39.73 & 39.79 & 38.40 \\
\hline $\mathrm{Al}_{2} \mathrm{O}_{3}$ & 0.00 & 0.00 & 0.02 & 0.03 & 0.00 & 0.01 & 0.00 & 0.00 & 0.00 & 0.00 & 0.01 & 0.00 & 0.00 \\
\hline $\mathrm{TiO}_{2}$ & 0.00 & 0.00 & 0.00 & 0.01 & 0.01 & 0.01 & 0.02 & 0.00 & 0.04 & 0.04 & 0.01 & 0.01 & 0.02 \\
\hline $\mathrm{FeO}^{2}$ & 24.99 & 26.17 & 30.10 & 31.72 & 24.88 & 26.56 & 27.85 & 20.73 & 22.49 & 24.29 & 15.79 & 17.41 & 22.38 \\
\hline $\mathrm{MnO}$ & 0.45 & 0.36 & 0.54 & 0.54 & 0.38 & 0.44 & 0.44 & 0.31 & 0.41 & 0.33 & 0.25 & 0.31 & 0.46 \\
\hline $\mathrm{NiO}$ & 0.10 & 0.11 & 0.05 & 0.11 & 0.06 & 0.15 & 0.09 & 0.12 & 0.14 & 0.15 & 0.24 & 0.17 & 0.23 \\
\hline $\mathrm{MgO}$ & 36.33 & 35.51 & 32.07 & 31.85 & 37.83 & 35.27 & 34.70 & 40.07 & 38.26 & 38.39 & 43.71 & 43.24 & 39.13 \\
\hline $\mathrm{CaO}$ & 0.09 & 0.06 & 0.04 & 0.05 & 0.02 & 0.07 & 0.05 & 0.08 & 0.04 & 0.05 & 0.05 & 0.03 & 0.04 \\
\hline $\begin{array}{l}\text { Total } \\
\text { Atomic no. }\end{array}$ & $\begin{array}{c}99.88 \\
(\mathrm{O}=4)\end{array}$ & 99.85 & 99.87 & 100.99 & 101.61 & 100.21 & 100.82 & 99.94 & 99.51 & 100.48 & 99.78 & 100.95 & 100.64 \\
\hline $\mathrm{Si}$ & 1.003 & 1.001 & 1.004 & 0.992 & 0.996 & 1.001 & 1.000 & 0.999 & 1.000 & 0.977 & 1.005 & 1.002 & 0.996 \\
\hline Al & 0.000 & 0.000 & 0.001 & 0.001 & 0.000 & 0.000 & 0.000 & 0.000 & 0.000 & 0.000 & 0.000 & 0.000 & 0.000 \\
\hline $\mathrm{Ti}$ & 0.000 & 0.000 & 0.000 & 0.000 & 0.000 & 0.000 & 0.000 & 0.000 & 0.001 & 0.001 & 0.000 & 0.000 & 0.000 \\
\hline $\mathrm{Fe}$ & 0.552 & 0.582 & 0.682 & 0.717 & 0.539 & 0.590 & 0.618 & 0.448 & 0.493 & 0.533 & 0.334 & 0.366 & 0.485 \\
\hline $\mathrm{Mn}$ & 0.010 & 0.008 & 0.012 & 0.012 & 0.008 & 0.010 & 0.010 & 0.007 & 0.009 & 0.007 & 0.005 & 0.007 & 0.010 \\
\hline $\mathrm{Mg}$ & 1.430 & 1.406 & 1.295 & 1.283 & 1.460 & 1.396 & 1.371 & 1.544 & 1.495 & 1.502 & 1.648 & 1.622 & 1.512 \\
\hline $\mathrm{Ca}$ & 0.002 & 0.002 & 0.001 & 0.002 & 0.001 & 0.002 & 0.001 & 0.002 & 0.001 & 0.001 & 0.001 & 0.001 & 0.001 \\
\hline Total & 2.997 & 2.999 & 2.996 & 3.007 & 3.004 & 2.999 & 3.000 & 3.001 & 2.999 & 3.022 & 2.994 & 2.998 & 3.004 \\
\hline Fo & 72.1 & 70.7 & 65.5 & 64.1 & 73.0 & 70.3 & 68.9 & 77.5 & 75.2 & 73.8 & 83.1 & 81.6 & 75.7 \\
\hline
\end{tabular}

Notes: $\mathrm{Tr}=$ troctolite, $\mathrm{COGb}=$ clinopyroxene-olivine gabbro, $\mathrm{OCGb}=$ olivine-clinopyroxene gabbro, and $\mathrm{Gb}=$ gabbro. Analyses are reported in weight percent.

Table 3 (continued).

\begin{tabular}{|c|c|c|c|c|c|c|c|c|c|c|c|c|c|}
\hline Hole: & $923 \mathrm{~A}$ & $923 \mathrm{~A}$ & $923 \mathrm{~A}$ & $923 \mathrm{~A}$ & $923 \mathrm{~A}$ & $923 \mathrm{~A}$ & $923 \mathrm{~A}$ & $923 \mathrm{~A}$ & $923 \mathrm{~A}$ & $923 \mathrm{~A}$ & $923 \mathrm{~A}$ & $923 \mathrm{~A}$ & $923 \mathrm{~A}$ \\
\hline Core, section: & $7 R-2$ & $8 \mathrm{R}-2$ & $8 R-2$ & $8 \mathrm{R}-2$ & $8 \mathrm{R}-2$ & $9 \mathrm{R}-1$ & $9 \mathrm{R}-1$ & $9 \mathrm{R}-2$ & 9R-2 & $9 \mathrm{R}-2$ & $9 \mathrm{R}-2$ & 9R-3 & $9 R-3$ \\
\hline Piece no.: & 8 & 1 & 1 & 6 & 6 & 3 & 3 & 8 & 8 & 9 & 9 & 3 & $3 \mathrm{~B}$ \\
\hline Rock name: & COGb & $\operatorname{Tr}$ & $\operatorname{Tr}$ & COGb & COGb & OCGb & OCGb & $\mathrm{COGb}$ & $\mathrm{COGb}$ & OCGb & OCGb & OCGb & OCGb \\
\hline $\mathrm{SiO}_{2}$ & 37.65 & 39.33 & 39.50 & 38.30 & 38.03 & 38.19 & 37.89 & 39.78 & 38.51 & 38.09 & 38.11 & 37.99 & 37.59 \\
\hline $\mathrm{Al}_{2} \mathrm{O}_{3}$ & 0.00 & 0.01 & $\begin{array}{r}39.30 \\
0.01\end{array}$ & $\begin{array}{r}50.30 \\
0.00\end{array}$ & $\begin{array}{r}30.03 \\
0.00\end{array}$ & $\begin{array}{r}38.19 \\
0.03\end{array}$ & $\begin{array}{r}31.89 \\
0.02\end{array}$ & $\begin{array}{r}39.78 \\
0.03\end{array}$ & $\begin{array}{r}38.01 \\
0.00\end{array}$ & $\begin{array}{r}38.09 \\
0.00\end{array}$ & $\begin{array}{r}38.11 \\
0.00\end{array}$ & $\begin{array}{r}37.99 \\
0.00\end{array}$ & $\begin{array}{r}37.39 \\
0.00\end{array}$ \\
\hline $\mathrm{TiO}_{2}$ & 0.02 & 0.00 & 0.01 & 0.00 & 0.00 & 0.00 & 0.00 & 0.11 & 0.00 & 0.08 & 0.02 & 0.00 & 0.00 \\
\hline $\mathrm{FeO}^{2}$ & 23.48 & 19.90 & 21.20 & 20.54 & 21.65 & 21.91 & 22.60 & 20.72 & 21.32 & 24.06 & 24.97 & 24.56 & 25.92 \\
\hline $\mathrm{MnO}$ & 0.39 & 0.27 & 0.34 & 0.45 & 0.36 & 0.31 & 0.33 & 0.29 & 0.30 & 0.31 & 0.38 & 0.40 & 0.35 \\
\hline $\mathrm{NiO}$ & 0.22 & 0.09 & 0.10 & 0.06 & 0.07 & 0.01 & 0.03 & 0.05 & 0.12 & 0.10 & 0.08 & 0.11 & 0.12 \\
\hline $\mathrm{MgO}$ & 38.14 & 41.31 & 40.24 & 39.66 & 39.52 & 39.39 & 38.97 & 40.80 & 39.87 & 37.63 & 36.91 & 37.27 & 36.13 \\
\hline $\mathrm{CaO}$ & 0.03 & 0.04 & 0.04 & 0.06 & 0.07 & 0.04 & 0.01 & 0.01 & 0.05 & 0.01 & 0.03 & 0.04 & 0.05 \\
\hline $\begin{array}{l}\text { Total } \\
\text { Atomic no. }\end{array}$ & 99.94 & 100.94 & 101.44 & 99.07 & 99.68 & $\begin{array}{c}99.88 \\
(\mathrm{O}=4)\end{array}$ & 99.86 & 101.79 & 100.18 & 100.28 & 100.50 & 100.36 & 100.17 \\
\hline $\mathrm{Si}$ & 0.990 & 1.001 & 1.006 & 0.999 & 0.991 & 0.994 & 0.990 & 1.006 & 0.997 & 0.997 & 1.000 & 0.997 & 0.995 \\
\hline Al & 0.000 & 0.000 & 0.000 & 0.000 & 0.000 & 0.001 & 0.001 & 0.001 & 0.000 & 0.000 & 0.000 & 0.000 & 0.000 \\
\hline $\mathrm{Ti}$ & 0.000 & 0.000 & 0.000 & 0.000 & 0.000 & 0.000 & 0.000 & 0.002 & 0.000 & 0.002 & 0.000 & 0.000 & 0.000 \\
\hline $\mathrm{Fe}$ & 0.516 & 0.424 & 0.452 & 0.448 & 0.472 & 0.477 & 0.494 & 0.438 & 0.461 & 0.527 & 0.548 & 0.539 & 0.574 \\
\hline $\mathrm{Mn}$ & 0.009 & 0.006 & 0.007 & 0.010 & 0.008 & 0.007 & 0.007 & 0.006 & 0.007 & 0.007 & 0.008 & 0.009 & 0.008 \\
\hline $\mathrm{Mg}$ & 1.494 & 1.567 & 1.527 & 1.542 & 1.535 & 1.527 & 1.517 & 1.538 & 1.537 & 1.468 & 1.443 & 1.457 & 1.426 \\
\hline $\mathrm{Ca}$ & 0.001 & 0.001 & 0.001 & 0.002 & 0.002 & 0.001 & 0.000 & 0.000 & 0.001 & 0.000 & 0.001 & 0.001 & 0.001 \\
\hline Total & 3.010 & 2.999 & 2.994 & 3.001 & 3.009 & 3.006 & 3.010 & 2.992 & 3.003 & 3.001 & 3.000 & 3.003 & 3.005 \\
\hline Fo & 74.3 & 78.72 & 77.18 & 77.5 & 76.5 & 76.2 & 75.4 & 77.82 & 76.92 & 73.6 & 72.5 & 73.0 & 71.3 \\
\hline
\end{tabular}

Table 3 (continued).

\begin{tabular}{|c|c|c|c|c|c|c|c|c|c|c|c|c|c|}
\hline Hole: & $923 \mathrm{~A}$ & $923 \mathrm{~A}$ & $923 \mathrm{~A}$ & $923 \mathrm{~A}$ & $923 \mathrm{~A}$ & $923 \mathrm{~A}$ & $923 \mathrm{~A}$ & $923 \mathrm{~A}$ & $923 \mathrm{~A}$ & $923 \mathrm{~A}$ & $923 \mathrm{~A}$ & $921 \mathrm{E}$ & $921 \mathrm{E}$ \\
\hline Core, section: & $10 \mathrm{R}-2$ & $10 \mathrm{R}-3$ & $10 \mathrm{R}-3$ & $11 \mathrm{R}-1$ & $11 \mathrm{R}-2$ & $11 \mathrm{R}-2$ & $12 \mathrm{R}-1$ & $12 \mathrm{R}-1$ & $12 \mathrm{R}-1$ & $12 \mathrm{R}-1$ & $13 R-1$ & 2R-1 & $2 \mathrm{R}-1$ \\
\hline Piece no.: & 2 & 1 & 1 & 12 & 3 & 3 & $4 \mathrm{~B}$ & $4 B$ & $4 \mathrm{~B}$ & 4B & 6 & 6 & 6 \\
\hline Rock name: & OCGb & OCGb & OCGb & OCGb & OCGb & OCGb & $\operatorname{Tr}$ & $\operatorname{Tr}$ & $\mathrm{COGb}$ & $\mathrm{COGb}$ & COGb & OCGb & OCGb \\
\hline $\mathrm{SiO}_{2}$ & 38.59 & 37.62 & 37.15 & 37.25 & 38.10 & 37.70 & 39.67 & 39.86 & 39.76 & 39.68 & 39.43 & 38.14 & 37.70 \\
\hline $\mathrm{Al}_{2} \mathrm{O}_{3}$ & 0.01 & $\begin{array}{r}37.02 \\
0.00\end{array}$ & 0.02 & 0.03 & 0.02 & 0.00 & 0.00 & 0.02 & 0.00 & 0.01 & 0.01 & 0.00 & 0.00 \\
\hline $\mathrm{TiO}_{2}$ & 0.01 & 0.01 & 0.01 & 0.01 & 0.04 & 0.00 & 0.07 & 0.05 & 0.07 & 0.06 & 0.02 & 0.03 & 0.02 \\
\hline $\mathrm{FeO}^{2}$ & 24.67 & 25.35 & 30.76 & 23.86 & 23.83 & 26.67 & 14.83 & 15.39 & 14.03 & 14.24 & 17.76 & 25.20 & 26.11 \\
\hline $\mathrm{MnO}$ & $\begin{array}{r}24.07 \\
0.29\end{array}$ & 0.47 & 0.49 & $\begin{array}{r}23.00 \\
0.32\end{array}$ & 0.45 & 0.43 & 0.29 & 0.22 & 0.19 & 0.28 & 0.29 & 0.41 & 0.31 \\
\hline $\mathrm{NiO}$ & 0.12 & 0.10 & 0.07 & 0.07 & 0.01 & 0.00 & 0.28 & 0.11 & 0.24 & 0.17 & 0.22 & 0.07 & 0.05 \\
\hline $\mathrm{MgO}$ & 37.83 & 36.58 & 32.40 & 37.36 & 37.50 & 35.48 & 44.32 & 44.71 & 45.34 & 45.19 & 42.54 & 36.78 & 36.05 \\
\hline $\mathrm{CaO}$ & 0.05 & 0.03 & 0.02 & 0.06 & 0.04 & 0.05 & 0.06 & 0.05 & 0.07 & 0.04 & $\begin{array}{r}42.34 \\
0.06\end{array}$ & $\begin{array}{r}0.05 \\
0.70\end{array}$ & 0.07 \\
\hline $\begin{array}{l}\text { Total } \\
\text { Atomic no. }\end{array}$ & 101.57 & 100.15 & 100.93 & 98.96 & 99.99 & 100.32 & 99.52 & 100.41 & 99.70 & 99.66 & $\begin{array}{l}100.33 \\
(O=4)\end{array}$ & 100.67 & 100.30 \\
\hline $\mathrm{Si}$ & 0.999 & 0.994 & 0.999 & 0.990 & 0.999 & 0.999 & 1.003 & 0.999 & 0.999 & 0.998 & 1.002 & 1.000 & 0.997 \\
\hline $\mathrm{Al}$ & 0.000 & 0.000 & 0.001 & 0.001 & 0.001 & 0.000 & 0.000 & 0.001 & 0.000 & 0.000 & 0.000 & 0.000 & 0.000 \\
\hline $\mathrm{Ti}$ & 0.000 & 0.000 & 0.000 & 0.000 & 0.001 & 0.000 & 0.001 & 0.001 & 0.001 & 0.001 & 0.000 & 0.001 & 0.000 \\
\hline $\mathrm{Fe}$ & 0.534 & 0.560 & 0.692 & 0.530 & 0.523 & 0.591 & 0.314 & 0.323 & 0.295 & 0.300 & 0.377 & 0.552 & 0.577 \\
\hline Mn & 0.006 & 0.010 & 0.011 & 0.007 & 0.010 & 0.010 & 0.006 & 0.005 & 0.004 & 0.006 & 0.006 & 0.009 & 0.007 \\
\hline $\mathrm{Mg}$ & 1.459 & 1.440 & 1.298 & 1.479 & 1.465 & 1.400 & 1.670 & 1.670 & 1.698 & 1.694 & 1.610 & 1.437 & 1.420 \\
\hline $\mathrm{Ca}$ & 0.001 & 0.001 & 0.001 & 0.002 & 0.001 & 0.001 & 0.002 & 0.001 & 0.002 & 0.001 & 0.002 & 0.001 & 0.002 \\
\hline Total & 3.000 & 3.006 & 3.001 & 3.010 & 3.000 & 3.001 & 2.996 & 3.000 & 2.999 & 3.000 & 2.998 & 3.000 & 3.003 \\
\hline Fo & 73.2 & 72.0 & 65.2 & 73.6 & 73.7 & 70.3 & 84.19 & 83.81 & 85.20 & 84.97 & 81.0 & 72.2 & 71.1 \\
\hline
\end{tabular}


Table 3 (continued).

\begin{tabular}{lrrr}
\hline Hole: & $921 \mathrm{E}$ & $921 \mathrm{E}$ & $921 \mathrm{E}$ \\
Core, section: & $4 \mathrm{R}-1$ & \multicolumn{1}{c}{$7 \mathrm{R}-1$} & \multicolumn{1}{c}{$7 \mathrm{R}-1$} \\
Piece no.: & \multicolumn{1}{c}{5} & \multicolumn{1}{c}{$9 \mathrm{~B}$} & \multicolumn{1}{c}{$9 \mathrm{~B}$} \\
Rock name: & $\mathrm{OCGb}$ & \multicolumn{1}{c}{ OCGb } & \multicolumn{1}{c}{ OCGb } \\
\hline $\mathrm{SiO}_{2}$ & 38.47 & 38.63 & 38.50 \\
$\mathrm{Al}_{2} \mathrm{O}_{3}$ & 0.00 & 0.00 & 0.01 \\
$\mathrm{TiO}_{2}$ & 0.03 & 0.03 & 0.06 \\
$\mathrm{FeO}$ & 23.92 & 21.13 & 24.01 \\
$\mathrm{MnO}$ & 0.26 & 0.29 & 0.41 \\
$\mathrm{NiO}$ & 0.06 & 0.16 & 0.11 \\
$\mathrm{MgO}$ & 37.87 & 40.08 & 37.47 \\
$\mathrm{CaO}$ & 0.04 & 0.03 & 0.04 \\
$\mathrm{Total}$ & 100.64 & 100.36 & 100.59 \\
$\mathrm{Atomic}$ no. & & & \\
$\mathrm{Si}$ & 1.001 & 0.997 & 1.004 \\
$\mathrm{Al}$ & 0.000 & 0.000 & 0.000 \\
$\mathrm{Ti}$ & 0.001 & 0.001 & 0.001 \\
$\mathrm{Fe}$ & 0.521 & 0.456 & 0.524 \\
$\mathrm{Mn}$ & 0.006 & 0.006 & 0.009 \\
$\mathrm{Mg}$ & 1.469 & 1.541 & 1.456 \\
$\mathrm{Ca}$ & 0.001 & 0.001 & 0.001 \\
$\mathrm{Total}$ & 2.998 & 3.002 & 2.995 \\
$\mathrm{Fo}$ & 73.8 & 77.2 & 73.5 \\
\hline
\end{tabular}

Table 4. Representative microprobe analyses of primary clinopyroxene for gabbroic rocks from Holes 921E and 923A.

\begin{tabular}{|c|c|c|c|c|c|c|c|c|c|c|c|c|c|c|}
\hline Hole: & $923 \mathrm{~A}$ & $923 \mathrm{~A}$ & $923 \mathrm{~A}$ & $923 \mathrm{~A}$ & $923 \mathrm{~A}$ & $923 \mathrm{~A}$ & $923 \mathrm{~A}$ & $923 \mathrm{~A}$ & $923 \mathrm{~A}$ & $923 \mathrm{~A}$ & $923 \mathrm{~A}$ & $923 \mathrm{~A}$ & $923 \mathrm{~A}$ & $923 \mathrm{~A}$ \\
\hline Core, section: & $2 \mathrm{R}-1$ & $2 \mathrm{R}-1$ & $3 R-2$ & $3 R-2$ & $5 \mathrm{R}-1$ & $5 R-1$ & $5 R-3$ & $5 R-3$ & 7R-1 & 7R-1 & $7 R-2$ & $7 R-2$ & $7 \mathrm{R}-2$ & $8 \mathrm{R}-2$ \\
\hline Piece no.: & 3 & 3 & 1 & 1 & $2 \mathrm{~A}$ & $2 \mathrm{~A}$ & 1 & 1 & $2 \mathrm{~B}$ & $2 \mathrm{~B}$ & 1 & 1 & 8 & 1 \\
\hline Rock name: & $\mathrm{Gb}$ & $\mathrm{Gb}$ & $\mathrm{Gb}$ & Gb & $\mathrm{Gb}$ & $\mathrm{Gb}$ & $\mathrm{Gb}$ & $\mathrm{Gb}$ & $\mathrm{COGb}$ & COGb & $\mathrm{COGb}$ & $\mathrm{COGb}$ & $\mathrm{COGb}$ & $\operatorname{Tr}$ \\
\hline $\mathrm{SiO}_{2}$ & 51.61 & 51.64 & 52.30 & 51.85 & 52.80 & 52.57 & 51.84 & 51.59 & 51.49 & 51.53 & 50.81 & 51.09 & 51.62 & 52.76 \\
\hline $\mathrm{TiO}_{2}$ & 0.83 & 0.93 & 0.57 & 0.80 & 0.57 & 0.61 & 0.66 & 0.96 & 0.60 & 0.96 & 0.54 & 1.31 & 0.68 & 0.69 \\
\hline $\mathrm{Al}_{2} \mathrm{O}_{3}$ & 2.57 & 2.60 & 1.79 & 2.36 & 2.51 & 2.53 & 2.20 & 2.53 & 3.12 & 2.61 & 3.88 & 3.47 & 2.62 & 2.49 \\
\hline $\mathrm{Cr}_{2} \mathrm{O}_{3}$ & 0.19 & 0.15 & 0.00 & 0.00 & 0.00 & 0.00 & 0.04 & 0.06 & 0.56 & 0.13 & 1.30 & 0.60 & 0.38 & 0.11 \\
\hline $\mathrm{FeO}$ & 6.86 & 7.71 & 7.33 & 7.98 & 6.30 & 7.58 & 7.76 & 7.44 & 5.87 & 7.20 & 4.62 & 5.29 & 6.85 & 5.98 \\
\hline $\mathrm{MnO}$ & 0.13 & 0.23 & 0.16 & 0.17 & 0.21 & 0.32 & 0.21 & 0.22 & 0.22 & 0.21 & 0.07 & 0.25 & 0.21 & 0.14 \\
\hline $\mathrm{NiO}$ & 0.18 & 0.13 & 0.00 & 0.00 & 0.00 & 0.00 & 0.00 & 0.00 & 0.08 & 0.06 & 0.08 & 0.00 & 0.04 & 0.19 \\
\hline $\mathrm{MgO}$ & 15.28 & 15.57 & 15.04 & 14.84 & 16.12 & 16.97 & 15.21 & 15.14 & 15.81 & 15.03 & 16.00 & 15.50 & 15.04 & 15.47 \\
\hline $\mathrm{CaO}$ & 20.74 & 20.13 & 20.84 & 20.52 & 21.17 & 19.16 & 20.64 & 20.32 & 20.79 & 20.59 & 20.78 & 21.65 & 20.99 & 21.68 \\
\hline $\mathrm{Na}_{2} \mathrm{O}$ & 0.47 & 0.41 & 0.36 & 0.44 & 0.31 & 0.31 & 0.35 & 0.38 & 0.44 & 0.44 & 0.45 & 0.41 & 0.40 & 0.41 \\
\hline $\mathrm{K}_{2} \mathrm{O}$ & 0.00 & 0.00 & 0.00 & 0.00 & 0.00 & 0.00 & 0.00 & 0.00 & 0.01 & 0.00 & 0.00 & 0.00 & 0.00 & 0.00 \\
\hline Total & 98.85 & 99.48 & 98.40 & 98.95 & 99.98 & 100.05 & 98.92 & 98.62 & 99.00 & 98.76 & 98.52 & 99.57 & 98.83 & 99.90 \\
\hline Atomic no. & $(\mathrm{O}=6)$ & & & & & & & & & & & & & \\
\hline $\mathrm{Si}$ & 1.932 & 1.923 & 1.961 & 1.939 & 1.939 & 1.932 & 1.940 & 1.932 & 1.921 & 1.930 & 1.908 & 1.897 & 1.934 & 1.945 \\
\hline $\mathrm{Ti}$ & 0.023 & 0.026 & 0.016 & 0.022 & 0.016 & 0.017 & 0.019 & 0.027 & 0.017 & 0.027 & 0.015 & 0.037 & 0.019 & 0.019 \\
\hline $\mathrm{Al}$ & 0.113 & 0.114 & 0.079 & 0.104 & 0.109 & 0.110 & 0.097 & 0.111 & 0.137 & 0.115 & 0.172 & 0.152 & 0.116 & 0.108 \\
\hline $\mathrm{Cr}$ & 0.006 & 0.004 & 0.000 & 0.000 & 0.000 & 0.000 & 0.001 & 0.002 & 0.017 & 0.004 & 0.039 & 0.018 & 0.011 & 0.003 \\
\hline $\mathrm{Fe}$ & 0.215 & 0.240 & 0.230 & 0.249 & 0.194 & 0.233 & 0.243 & 0.233 & 0.183 & 0.225 & 0.145 & 0.164 & 0.215 & 0.184 \\
\hline Mn & 0.004 & 0.007 & 0.005 & 0.005 & 0.006 & 0.010 & 0.007 & 0.007 & 0.007 & 0.007 & 0.002 & 0.008 & 0.007 & 0.004 \\
\hline $\mathrm{Mg}$ & 0.852 & 0.864 & 0.841 & 0.827 & 0.882 & 0.929 & 0.848 & 0.845 & 0.880 & 0.839 & 0.895 & 0.858 & 0.840 & 0.850 \\
\hline $\mathrm{Ca}$ & 0.832 & 0.804 & 0.838 & 0.823 & 0.834 & 0.755 & 0.828 & 0.816 & 0.832 & 0.827 & 0.837 & 0.862 & 0.843 & 0.857 \\
\hline $\mathrm{Na}$ & 0.034 & 0.030 & 0.026 & 0.032 & 0.022 & 0.022 & 0.026 & 0.028 & 0.032 & 0.032 & 0.033 & 0.030 & 0.029 & 0.029 \\
\hline $\mathrm{K}$ & 0.000 & 0.000 & 0.000 & 0.000 & 0.000 & 0.000 & 0.000 & 0.000 & 0.000 & 0.000 & 0.000 & 0.000 & 0.000 & 0.000 \\
\hline Total & 4.011 & 4.013 & 3.996 & 4.002 & 4.002 & 4.007 & 4.007 & 4.001 & 4.026 & 4.005 & 4.046 & 4.024 & 4.014 & 4.000 \\
\hline $\mathrm{Mg} \#$ & 79.9 & 78.3 & 78.5 & 76.8 & 82.0 & 80.0 & 77.7 & 78.4 & 82.8 & 78.8 & 86.0 & 83.9 & 79.7 & 82.2 \\
\hline $\mathrm{Ca} /(\mathrm{Ca}+\mathrm{Mg}+\mathrm{Fe})$ & 43.8 & 42.1 & 43.9 & 43.3 & 43.7 & 39.4 & 43.1 & 43.1 & 43.9 & 43.7 & 44.6 & 45.7 & 44.4 & 45.3 \\
\hline $\mathrm{Mg} /(\mathrm{Ca}+\mathrm{Mg}+\mathrm{Fe})$ & 44.9 & 45.3 & 44.1 & 43.6 & 46.2 & 48.5 & 44.2 & 44.6 & 46.4 & 44.4 & 47.7 & 45.5 & 44.3 & 44.9 \\
\hline $\mathrm{Fe} /(\mathrm{Ca}+\mathrm{Mg}+\mathrm{Fe})$ & 11.3 & 12.6 & 12.0 & 13.1 & 10.1 & 12.2 & 12.7 & 12.3 & 9.7 & 11.9 & 7.7 & 8.7 & 11.3 & 9.8 \\
\hline
\end{tabular}

Notes: $\mathrm{Tr}=$ troctolite, $\mathrm{COG} b=$ clinopyroxene-olivine gabbro, $\mathrm{OCG} b=$ olivine-clinopyroxene gabbro, $\mathrm{Gb}=$ gabbro, $\mathrm{GbN}=$ gabbronorite, $\mathrm{OxGb}=\mathrm{oxide}$ gabbro, and $\mathrm{QzDi}=$ quartz diorite. Analyses are reported in weight percent. 
Table 4 (continued).

\begin{tabular}{|c|c|c|c|c|c|c|c|c|c|c|c|c|c|c|}
\hline Hole: & $923 \mathrm{~A}$ & $923 \mathrm{~A}$ & $923 \mathrm{~A}$ & $923 \mathrm{~A}$ & $923 \mathrm{~A}$ & $923 \mathrm{~A}$ & $923 \mathrm{~A}$ & $923 \mathrm{~A}$ & $923 \mathrm{~A}$ & $923 \mathrm{~A}$ & $923 \mathrm{~A}$ & $923 \mathrm{~A}$ & $923 \mathrm{~A}$ & $923 \mathrm{~A}$ \\
\hline Core, section: & $8 \mathrm{R}-2$ & $9 \mathrm{R}-1$ & $9 \mathrm{R}-2$ & $9 \mathrm{R}-2$ & $9 \mathrm{R}-2$ & $9 \mathrm{R}-2$ & $9 \mathrm{R}-3$ & $9 \mathrm{R}-3$ & $10 \mathrm{R}-2$ & $10 \mathrm{R}-2$ & $10 \mathrm{R}-3$ & $11 \mathrm{R}-1$ & $11 R-1$ & $11 \mathrm{R}-1$ \\
\hline Piece no.: & 1 & $3 \mathrm{D}$ & 8 & 8 & 9 & 9 & 3B & 3B & 2 & 2 & 1 & 12 & 12 & 12 \\
\hline Rock name: & $\operatorname{Tr}$ & OCGb & $\mathrm{COGb}$ & COGb & OCGb & OCGb & OCGb & OCGb & OCGb & OCGb & OCGb & OCGb & OCGb & OCGb \\
\hline $\mathrm{SiO}_{2}$ & 52.57 & 51.60 & 51.94 & 52.21 & 52.22 & 52.09 & 52.51 & 52.74 & 52.50 & 51.99 & 52.52 & 52.56 & 52.74 & 52.17 \\
\hline $\mathrm{TiO}_{2}$ & 0.86 & 0.58 & 1.26 & 0.70 & 0.51 & 0.89 & 0.49 & 0.49 & 0.39 & 0.78 & 0.59 & 0.52 & 0.48 & 0.48 \\
\hline $\mathrm{Al}_{2} \mathrm{O}_{3}$ & 2.54 & 2.65 & 3.46 & 2.29 & 2.85 & 2.66 & 2.79 & 3.16 & 2.20 & 1.89 & 2.16 & 2.69 & 2.91 & 2.62 \\
\hline $\mathrm{Cr}_{2} \mathrm{O}_{3}$ & 0.18 & 0.12 & 0.14 & 0.06 & 0.21 & 0.06 & 0.14 & 0.28 & 0.03 & 0.09 & 0.05 & 0.14 & 0.18 & 0.17 \\
\hline $\mathrm{FeO}$ & 6.51 & 6.23 & 5.71 & 5.50 & 5.67 & 6.58 & 5.80 & 6.93 & 7.26 & 7.63 & 6.98 & 5.61 & 6.63 & 7.83 \\
\hline $\mathrm{MnO}$ & 0.11 & 0.22 & 0.13 & 0.17 & 0.13 & 0.15 & 0.16 & 0.17 & 0.13 & 0.26 & 0.17 & 0.18 & 0.17 & 0.18 \\
\hline $\mathrm{NiO}$ & 0.13 & 0.00 & 0.07 & 0.00 & 0.05 & 0.09 & 0.00 & 0.00 & 0.00 & 0.03 & 0.06 & 0.05 & 0.03 & 0.02 \\
\hline $\mathrm{MgO}$ & 15.85 & 15.72 & 15.30 & 15.63 & 16.92 & 15.30 & 16.60 & 16.55 & 16.55 & 16.20 & 17.17 & 16.20 & 16.74 & 17.74 \\
\hline $\mathrm{CaO}$ & 21.29 & 21.31 & 22.47 & 22.45 & 19.70 & 20.92 & 20.22 & 19.01 & 20.61 & 19.85 & 19.02 & 21.19 & 19.98 & 18.14 \\
\hline $\mathrm{Na}_{2} \mathrm{O}$ & 0.39 & 0.36 & 0.42 & 0.40 & 0.36 & 0.42 & 0.37 & 0.37 & 0.31 & 0.33 & 0.35 & 0.42 & 0.42 & 0.27 \\
\hline $\mathrm{K}_{2} \mathrm{O}$ & 0.00 & 0.00 & 0.00 & 0.00 & 0.00 & 0.00 & 0.00 & 0.00 & 0.00 & 0.00 & 0.00 & 0.00 & 0.00 & 0.00 \\
\hline $\begin{array}{l}\text { Total } \\
\text { Atomic no. }\end{array}$ & 100.42 & 98.79 & 100.90 & 99.40 & 98.60 & $\begin{array}{c}99.18 \\
(\mathrm{O}=6)\end{array}$ & 99.07 & 99.71 & 99.98 & 99.05 & 99.07 & 99.56 & 100.28 & 99.62 \\
\hline $\mathrm{Si}$ & 1.932 & 1.926 & 1.899 & 1.934 & 1.937 & 1.935 & 1.940 & 1.940 & 1.936 & 1.939 & 1.945 & 1.938 & 1.932 & 1.926 \\
\hline $\mathrm{Ti}$ & 0.024 & 0.016 & 0.035 & 0.019 & 0.014 & 0.025 & 0.014 & 0.014 & 0.011 & 0.022 & 0.016 & 0.014 & 0.013 & 0.013 \\
\hline $\mathrm{Al}$ & 0.110 & 0.116 & 0.149 & 0.100 & 0.125 & 0.117 & 0.121 & 0.137 & 0.096 & 0.083 & 0.094 & 0.117 & 0.125 & 0.114 \\
\hline $\mathrm{Cr}$ & 0.005 & 0.004 & 0.004 & 0.002 & 0.006 & 0.002 & 0.004 & 0.008 & 0.001 & 0.003 & 0.001 & 0.004 & 0.005 & 0.005 \\
\hline $\mathrm{Fe}$ & 0.200 & 0.194 & 0.175 & 0.170 & 0.176 & 0.205 & 0.179 & 0.213 & 0.224 & 0.238 & 0.216 & 0.173 & 0.203 & 0.242 \\
\hline Mn & 0.003 & 0.007 & 0.004 & 0.005 & 0.004 & 0.005 & 0.005 & 0.005 & 0.004 & 0.008 & 0.005 & 0.006 & 0.005 & 0.006 \\
\hline $\mathrm{Mg}$ & 0.868 & 0.874 & 0.834 & 0.863 & 0.935 & 0.847 & 0.914 & 0.907 & 0.909 & 0.901 & 0.948 & 0.890 & 0.914 & 0.976 \\
\hline $\mathrm{Ca}$ & 0.839 & 0.853 & 0.881 & 0.892 & 0.783 & 0.833 & 0.801 & 0.750 & 0.815 & 0.794 & 0.755 & 0.837 & 0.785 & 0.718 \\
\hline $\mathrm{Na}$ & 0.028 & 0.026 & 0.030 & 0.028 & 0.026 & 0.030 & 0.026 & 0.027 & 0.022 & 0.024 & 0.025 & 0.030 & 0.030 & 0.020 \\
\hline $\mathrm{K}$ & 0.000 & 0.000 & 0.000 & 0.000 & 0.000 & 0.000 & 0.000 & 0.000 & 0.000 & 0.000 & 0.000 & 0.000 & 0.000 & 0.000 \\
\hline Total & 4.009 & 4.016 & 4.010 & 4.013 & 4.006 & 3.999 & 4.003 & 4.000 & 4.018 & 4.012 & 4.006 & 4.009 & 4.012 & 4.019 \\
\hline Mg\# & 81.3 & 81.8 & 82.7 & 83.5 & 84.2 & 80.5 & 83.6 & 81.0 & 80.2 & 79.1 & 81.4 & 83.7 & 81.8 & 80.1 \\
\hline $\mathrm{Ca} /(\mathrm{Ca}+\mathrm{Mg}+\mathrm{Fe})$ & 44.0 & 44.4 & 46.6 & 46.3 & 41.4 & 44.2 & 42.3 & 40.1 & 41.8 & 41.1 & 39.3 & 44.1 & 41.3 & 37.1 \\
\hline $\mathrm{Mg} /(\mathrm{Ca}+\mathrm{Mg}+\mathrm{Fe})$ & 45.5 & 45.5 & 44.1 & 44.8 & 49.4 & 44.9 & 48.3 & 48.5 & 46.7 & 46.6 & 49.4 & 46.8 & 48.1 & 50.4 \\
\hline $\mathrm{Fe} /(\mathrm{Ca}+\mathrm{Mg}+\mathrm{Fe})$ & 10.5 & 10.1 & 9.2 & 8.8 & 9.3 & 10.9 & 9.5 & 11.4 & 11.5 & 12.3 & 11.3 & 9.1 & 10.7 & 12.5 \\
\hline
\end{tabular}

Table 4 (continued).

\begin{tabular}{|c|c|c|c|c|c|c|c|c|c|c|c|c|c|c|}
\hline Hole: & $923 \mathrm{~A}$ & $923 \mathrm{~A}$ & $923 \mathrm{~A}$ & $923 \mathrm{~A}$ & $923 \mathrm{~A}$ & $923 \mathrm{~A}$ & $923 \mathrm{~A}$ & $923 \mathrm{~A}$ & $923 \mathrm{~A}$ & $921 \mathrm{E}$ & $921 \mathrm{E}$ & $921 \mathrm{E}$ & $921 \mathrm{E}$ & $921 \mathrm{E}$ \\
\hline Core, section: & $11 R-2$ & $11 \mathrm{R}-2$ & $11 \mathrm{R}-2$ & $12 \mathrm{R}-1$ & $12 \mathrm{R}-1$ & $12 \mathrm{R}-1$ & $12 \mathrm{R}-1$ & $13 R-1$ & $13 \mathrm{R}-1$ & $2 \mathrm{R}-1$ & $2 R-1$ & $3 R-1$ & $3 \mathrm{R}-1$ & $4 \mathrm{R}-1$ \\
\hline Piece no.: & 3 & 3 & 3 & $4 B$ & 4B & $4 B$ & $4 B$ & 6 & 6 & 6 & 6 & 5 & 5 & 5 \\
\hline Rock name: & OCGb & OCGb & OCGb & $\operatorname{Tr}$ & $\operatorname{Tr}$ & $\mathrm{COGb}$ & $\mathrm{COGb}$ & COGb & $\mathrm{COGb}$ & OCGb & OCGb & OxGb & $\mathrm{OxGb}$ & $\mathrm{OCGb}$ \\
\hline $\mathrm{SiO}_{2}$ & 52.59 & 52.92 & 51.99 & 51.35 & 51.88 & 52.26 & 51.86 & 52.98 & 52.12 & 51.89 & 52.28 & 51.80 & 51.91 & 52.73 \\
\hline $\mathrm{TiO}_{2}^{2}$ & 0.46 & 0.40 & 0.78 & 1.27 & 1.11 & 0.26 & 0.52 & 0.72 & 0.70 & 0.47 & 0.46 & 0.76 & 0.69 & 0.49 \\
\hline $\mathrm{Al}_{2} \mathrm{O}_{3}$ & 2.90 & 2.34 & 2.60 & 3.37 & 3.20 & 3.20 & 3.82 & 1.89 & 2.99 & 2.66 & 2.59 & 2.33 & 1.88 & 2.70 \\
\hline $\mathrm{Cr}_{2} \mathrm{O}_{3}$ & 0.19 & 0.11 & 0.00 & 0.25 & 0.20 & 1.15 & 1.16 & 0.08 & 0.13 & 0.11 & 0.10 & 0.14 & 0.09 & 0.16 \\
\hline $\mathrm{FeO}$ & 5.32 & 6.49 & 7.58 & 3.69 & 4.48 & 3.76 & 3.87 & 4.18 & 5.23 & 5.52 & 5.53 & 10.02 & 10.51 & 4.96 \\
\hline $\mathrm{MnO}$ & 0.16 & 0.17 & 0.17 & 0.11 & 0.13 & 0.16 & 0.17 & 0.07 & 0.09 & 0.09 & 0.14 & 0.39 & 0.30 & 0.10 \\
\hline $\mathrm{NiO}$ & 0.00 & 0.00 & 0.00 & 0.00 & 0.00 & 0.00 & 0.00 & 0.17 & 0.19 & 0.03 & 0.00 & 0.00 & 0.00 & 0.05 \\
\hline $\mathrm{MgO}$ & 16.47 & 17.96 & 15.06 & 15.95 & 15.89 & 17.56 & 16.11 & 16.43 & 15.62 & 16.74 & 16.62 & 12.73 & 12.47 & 16.29 \\
\hline $\mathrm{CaO}$ & 20.91 & 18.65 & 20.53 & 23.09 & 21.98 & 20.36 & 21.71 & 22.14 & 21.34 & 20.89 & 20.71 & 20.52 & 20.75 & 21.08 \\
\hline $\mathrm{Na}_{2} \mathrm{O}$ & 0.37 & 0.36 & 0.40 & 0.38 & 0.43 & 0.41 & 0.50 & 0.28 & 0.41 & 0.42 & 0.33 & 0.46 & 0.48 & 0.39 \\
\hline $\mathrm{K}_{2} \mathrm{O}$ & 0.00 & 0.00 & 0.00 & 0.02 & 0.02 & 0.00 & 0.02 & 0.00 & 0.00 & 0.00 & 0.00 & 0.00 & 0.00 & 0.00 \\
\hline $\begin{array}{l}\text { Total } \\
\text { Atomic no. }\end{array}$ & 99.38 & 99.41 & 99.11 & 99.47 & 99.32 & 99.14 & 99.74 & 98.93 & 98.84 & 98.81 & $\begin{array}{c}98.76 \\
(\mathrm{O}=6)\end{array}$ & 99.14 & 99.06 & 98.95 \\
\hline $\mathrm{Si}$ & 1.937 & 1.945 & 1.937 & 1.894 & 1.914 & 1.933 & 1.916 & 1.957 & 1.935 & 1.926 & 1.938 & 1.955 & 1.965 & 1.948 \\
\hline Ti & 0.013 & 0.011 & 0.022 & 0.035 & 0.031 & 0.007 & 0.014 & 0.020 & 0.020 & 0.013 & 0.013 & 0.021 & 0.020 & 0.014 \\
\hline Al & 0.126 & 0.101 & 0.114 & 0.146 & 0.139 & 0.139 & 0.166 & 0.082 & 0.131 & 0.116 & 0.113 & 0.104 & 0.084 & 0.118 \\
\hline $\mathrm{Cr}$ & 0.006 & 0.003 & 0.000 & 0.007 & 0.006 & 0.034 & 0.034 & 0.002 & 0.004 & 0.003 & 0.003 & 0.004 & 0.003 & 0.005 \\
\hline $\mathrm{Fe}$ & 0.164 & 0.200 & 0.236 & 0.114 & 0.138 & 0.116 & 0.120 & 0.129 & 0.163 & 0.171 & 0.171 & 0.316 & 0.333 & 0.153 \\
\hline $\mathrm{Mn}$ & 0.005 & 0.005 & 0.005 & 0.003 & 0.004 & 0.005 & 0.005 & 0.002 & 0.003 & 0.003 & 0.004 & 0.012 & 0.010 & 0.003 \\
\hline $\mathrm{Mg}$ & 0.904 & 0.983 & 0.836 & 0.877 & 0.874 & 0.968 & 0.887 & 0.904 & 0.864 & 0.926 & 0.918 & 0.716 & 0.704 & 0.897 \\
\hline $\mathrm{Ca}$ & 0.826 & 0.735 & 0.820 & 0.913 & 0.869 & 0.807 & 0.860 & 0.877 & 0.850 & 0.831 & 0.823 & 0.830 & 0.842 & 0.835 \\
\hline $\mathrm{Na}$ & 0.026 & 0.026 & 0.029 & 0.027 & 0.031 & 0.030 & 0.036 & 0.020 & 0.030 & 0.030 & 0.024 & 0.033 & 0.035 & 0.028 \\
\hline $\mathrm{K}$ & 0.000 & 0.000 & 0.000 & 0.001 & 0.001 & 0.000 & 0.001 & 0.000 & 0.000 & 0.000 & 0.000 & 0.000 & 0.000 & 0.000 \\
\hline Total & 4.006 & 4.009 & 3.999 & 4.019 & 4.007 & 4.039 & 4.039 & 3.994 & 3.999 & 4.021 & 4.007 & 3.993 & 3.994 & 3.998 \\
\hline $\mathrm{Mg \#}$ & 84.7 & 83.1 & 78.0 & 88.5 & 86.3 & 89.3 & 88.1 & 87.5 & 84.2 & 84.4 & 84.3 & 69.4 & 67.9 & 85.4 \\
\hline $\mathrm{Ca} /(\mathrm{Ca}+\mathrm{Mg}+\mathrm{Fe})$ & 43.6 & 38.3 & 43.3 & 48.0 & 46.2 & 42.7 & 46.1 & 45.9 & 45.3 & 43.1 & 43.0 & 44.6 & 44.8 & 44.3 \\
\hline $\mathrm{Mg} /(\mathrm{Ca}+\mathrm{Mg}+\mathrm{Fe})$ & 47.8 & 51.3 & 44.2 & 46.1 & 46.4 & 51.2 & 47.5 & 47.3 & 46.1 & 48.0 & 48.0 & 38.4 & 37.5 & 47.6 \\
\hline $\mathrm{Fe} /(\mathrm{Ca}+\mathrm{Mg}+\mathrm{Fe})$ & 8.6 & 10.4 & 12.5 & 6.0 & 7.3 & 6.2 & 6.4 & 6.8 & 8.7 & 8.9 & 9.0 & 17.0 & 17.7 & 8.1 \\
\hline
\end{tabular}


Table 5. Representative microprobe analyses of primary orthopyroxene for gabbroic rocks from Holes 921E and 923A.

\begin{tabular}{|c|c|c|c|c|c|c|c|c|c|c|c|c|c|c|c|c|c|c|}
\hline Hole: & $923 \mathrm{~A}$ & $923 \mathrm{~A}$ & $923 \mathrm{~A}$ & $923 \mathrm{~A}$ & $923 \mathrm{~A}$ & $923 \mathrm{~A}$ & $923 \mathrm{~A}$ & $923 \mathrm{~A}$ & $923 \mathrm{~A}$ & $923 \mathrm{~A}$ & $923 \mathrm{~A}$ & $921 \mathrm{E}$ & $921 \mathrm{E}$ & $921 \mathrm{E}$ & $921 \mathrm{E}$ & $921 \mathrm{E}$ & $921 \mathrm{E}$ & $921 \mathrm{E}$ \\
\hline Core, section: & $3 \mathrm{R}-2$ & $3 \mathrm{R}-2$ & $5 \mathrm{R}-1$ & $5 \mathrm{R}-3$ & $5 R-3$ & $8 \mathrm{R}-2$ & $8 \mathrm{R}-2$ & 9R-1 & $9 \mathrm{R}-2$ & $12 \mathrm{R}-1$ & $12 \mathrm{R}-1$ & $3 R-1$ & $7 \mathrm{R}-1$ & $7 \mathrm{R}-1$ & $8 \mathrm{R}-1$ & $8 \mathrm{R}-1$ & $8 \mathrm{R}-1$ & $8 \mathrm{R}-1$ \\
\hline Piece no.: & 1 & 1 & $2 \mathrm{~A}$ & 1 & 1 & 1 & 1 & 3D & 9 & 4B & 4B & 5 & 4 & 9B & 1 & 1 & $7 \mathrm{~B}$ & $7 \mathrm{~B}$ \\
\hline Rock name: & Gb & $\mathrm{Gb}$ & $\mathrm{Gb}$ & $\mathrm{Gb}$ & Gb & $\operatorname{Tr}$ & $\operatorname{Tr}$ & OCGb & OCGb & $\operatorname{Tr}$ & COGb & OxGb & QzDi & OCGb & QzDi & $\mathrm{QzDi}$ & QzDi & $\mathrm{GbN}$ \\
\hline $\mathrm{SiO}_{2}$ & 53.58 & 53.12 & 54.79 & 53.36 & 54.17 & 55.26 & 55.17 & 53.85 & 54.20 & 56.23 & 56.13 & 51.94 & 53.38 & 55.30 & 54.44 & 54.36 & 53.92 & 53.45 \\
\hline $\mathrm{TiO}_{2}$ & 0.28 & 0.41 & 0.24 & 0.23 & 0.34 & 0.24 & 0.38 & 0.33 & 0.34 & 0.12 & 0.17 & 0.37 & 0.31 & 0.25 & 0.19 & 0.17 & 0.36 & 0.36 \\
\hline $\mathrm{Al}_{2} \mathrm{O}_{3}$ & 1.06 & 1.19 & 0.98 & 1.15 & 0.89 & 1.09 & 1.36 & 1.25 & 1.27 & 0.84 & 1.09 & 0.77 & 1.21 & 1.21 & 1.03 & 1.11 & 1.29 & 1.22 \\
\hline $\mathrm{Cr}_{2} \mathrm{O}_{3}$ & 0.00 & 0.00 & 0.00 & 0.00 & 0.02 & 0.00 & 0.07 & 0.02 & 0.01 & 0.00 & 0.14 & 0.02 & 0.00 & 0.04 & 0.05 & 0.04 & 0.04 & 0.06 \\
\hline $\mathrm{FeO}$ & 18.35 & 19.92 & 15.20 & 16.21 & 16.73 & 12.72 & 12.42 & 14.12 & 14.71 & 9.45 & 9.23 & 22.35 & 24.05 & 14.74 & 25.11 & 25.51 & 18.57 & 20.06 \\
\hline $\mathrm{MnO}$ & 0.49 & 0.46 & 0.45 & 0.38 & 0.45 & 0.23 & 0.33 & 0.37 & 0.37 & 0.30 & 0.31 & 0.61 & 0.80 & 0.26 & 0.86 & 0.89 & 0.42 & 0.49 \\
\hline $\mathrm{NiO}$ & 0.00 & 0.00 & 0.01 & 0.00 & 0.00 & 0.12 & 0.05 & 0.00 & 0.05 & 0.00 & 0.00 & 0.00 & 0.00 & 0.00 & 0.00 & 0.00 & 0.00 & 0.00 \\
\hline $\mathrm{MgO}$ & 25.13 & 23.71 & 28.09 & 26.99 & 26.49 & 29.44 & 29.17 & 28.55 & 27.25 & 31.45 & 31.73 & 20.06 & 15.86 & 27.64 & 16.46 & 16.25 & 24.67 & 23.23 \\
\hline $\mathrm{CaO}$ & 1.15 & 1.08 & 0.89 & 0.97 & 1.02 & 0.71 & 1.04 & 0.90 & 1.22 & 0.76 & 0.80 & 1.68 & 1.80 & 0.90 & 1.41 & 1.80 & 1.19 & 1.37 \\
\hline $\mathrm{Na}_{2} \mathrm{O}$ & 0.03 & 0.03 & 0.01 & 0.02 & 0.02 & 0.00 & 0.02 & 0.00 & 0.04 & 0.03 & 0.03 & 0.02 & 0.43 & 0.01 & 0.29 & 0.36 & 0.04 & 0.04 \\
\hline $\mathrm{K}_{2} \mathrm{O}$ & 0.00 & 0.00 & 0.00 & 0.00 & 0.00 & 0.00 & 0.00 & 0.00 & 0.00 & 0.02 & 0.00 & 0.00 & 0.00 & 0.00 & 0.00 & 0.00 & 0.00 & 0.00 \\
\hline $\begin{array}{l}\text { Total } \\
\text { Atomic no. }\end{array}$ & $\begin{array}{l}100.06 \\
(\mathrm{O}=6)\end{array}$ & 99.91 & 100.66 & 99.31 & 100.12 & 99.82 & 100.01 & 99.37 & 99.46 & 99.20 & 99.63 & 97.82 & 97.84 & 100.35 & 99.82 & 100.49 & 100.50 & 100.28 \\
\hline $\mathrm{Si}$ & 1.961 & 1.961 & 1.961 & 1.949 & 1.965 & 1.970 & 1.964 & 1.946 & 1.962 & 1.984 & 1.974 & 1.988 & 2.051 & 1.977 & 2.054 & 2.045 & 1.965 & 1.969 \\
\hline $\mathrm{Ti}$ & 0.008 & 0.011 & 0.007 & 0.006 & 0.009 & 0.006 & 0.010 & 0.009 & 0.009 & 0.003 & 0.004 & 0.011 & 0.009 & 0.007 & 0.005 & 0.005 & 0.010 & 0.010 \\
\hline $\mathrm{Al}$ & 0.046 & 0.052 & 0.041 & 0.050 & 0.038 & 0.046 & 0.057 & 0.053 & 0.054 & 0.035 & 0.045 & 0.035 & 0.055 & 0.051 & 0.046 & 0.049 & 0.056 & 0.053 \\
\hline $\mathrm{Cr}$ & 0.000 & 0.000 & 0.000 & 0.000 & 0.001 & 0.000 & 0.002 & 0.000 & 0.000 & 0.000 & 0.004 & 0.001 & 0.000 & 0.001 & 0.001 & 0.001 & 0.001 & 0.002 \\
\hline $\mathrm{Fe}$ & 0.562 & 0.615 & 0.455 & 0.495 & 0.507 & 0.379 & 0.370 & 0.427 & 0.445 & 0.279 & 0.272 & 0.715 & 0.773 & 0.441 & 0.792 & 0.802 & 0.566 & 0.618 \\
\hline Mn & 0.015 & 0.014 & 0.014 & 0.012 & 0.014 & 0.007 & 0.010 & 0.011 & 0.011 & 0.009 & 0.009 & 0.020 & 0.026 & 0.008 & 0.028 & 0.028 & 0.013 & 0.015 \\
\hline $\mathrm{Mg}$ & 1.371 & 1.304 & 1.499 & 1.469 & 1.432 & 1.564 & 1.547 & 1.538 & 1.470 & 1.654 & 1.663 & 1.145 & 0.908 & 1.473 & 0.925 & 0.911 & 1.340 & 1.275 \\
\hline $\mathrm{Ca}$ & 0.045 & 0.043 & 0.034 & 0.038 & 0.040 & 0.027 & 0.040 & 0.035 & 0.047 & 0.029 & 0.030 & 0.069 & 0.074 & 0.034 & 0.057 & 0.073 & 0.047 & 0.054 \\
\hline $\mathrm{Na}$ & 0.002 & 0.002 & 0.001 & 0.001 & 0.001 & 0.000 & 0.002 & 0.000 & 0.003 & 0.002 & 0.002 & 0.002 & 0.032 & 0.001 & 0.021 & 0.026 & 0.003 & 0.003 \\
\hline $\mathrm{K}$ & 0.000 & 0.000 & 0.000 & 0.000 & 0.000 & 0.000 & 0.000 & 0.000 & 0.000 & 0.001 & 0.000 & 0.000 & 0.000 & 0.000 & 0.000 & 0.000 & 0.000 & 0.000 \\
\hline Total & 4.009 & 4.003 & 4.012 & 4.020 & 4.008 & 4.000 & 4.000 & 4.019 & 4.003 & 3.996 & 4.004 & 3.985 & 3.929 & 3.993 & 3.930 & 3.940 & 4.000 & 3.998 \\
\hline Mg\# & 70.9 & 68.0 & 76.7 & 74.8 & 73.8 & 80.5 & 80.7 & 78.3 & 76.8 & 85.6 & 86.0 & 61.5 & 54.0 & 77.0 & 53.9 & 53.2 & 70.3 & 67.4 \\
\hline $\mathrm{Ca} /(\mathrm{Ca}+\mathrm{Mg}+\mathrm{Fe})$ & 2.3 & 2.2 & 1.7 & 1.9 & 2.0 & 1.4 & 2.0 & 1.7 & 2.4 & 1.5 & 1.5 & 3.6 & 4.2 & 1.8 & 3.2 & 4.1 & 2.4 & 2.8 \\
\hline $\mathrm{Mg} /(\mathrm{C}$ & 69.3 & 66.5 & 75.4 & 73.4 & 72.4 & 79.4 & 79.1 & 76.9 & 74.9 & 84.3 & 84.6 & 59.3 & 51.8 & 75.6 & 52.2 & 51.0 & 68.6 & 65.5 \\
\hline $\mathrm{Fe} /(\mathrm{Ca}+\mathrm{Mg}+\mathrm{Fe})$ & 28.4 & 31.3 & 22.9 & 24.7 & 25.6 & 19.2 & 18.9 & 21.3 & 22.7 & 14.2 & 13.8 & 37.1 & 44.0 & 22.6 & 44.6 & 44.9 & 29.0 & 31.7 \\
\hline
\end{tabular}

Notes: $\mathrm{Tr}=$ troctolite, $\mathrm{COGb}=$ clinopyroxene-olivine gabbro, $\mathrm{OCGb}=$ olivine-clinopyroxene gabbro, $\mathrm{Gb}=$ gabbro, $\mathrm{GbN}=$ gabbronorite, $\mathrm{OxGb}=$ oxide gabbro, and $\mathrm{QzDi}=$ quartz diorite. Analyses are reported in weight percent. 


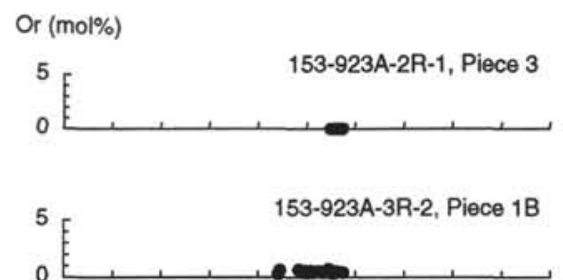

$$
\text { Or (mol\%) }
$$
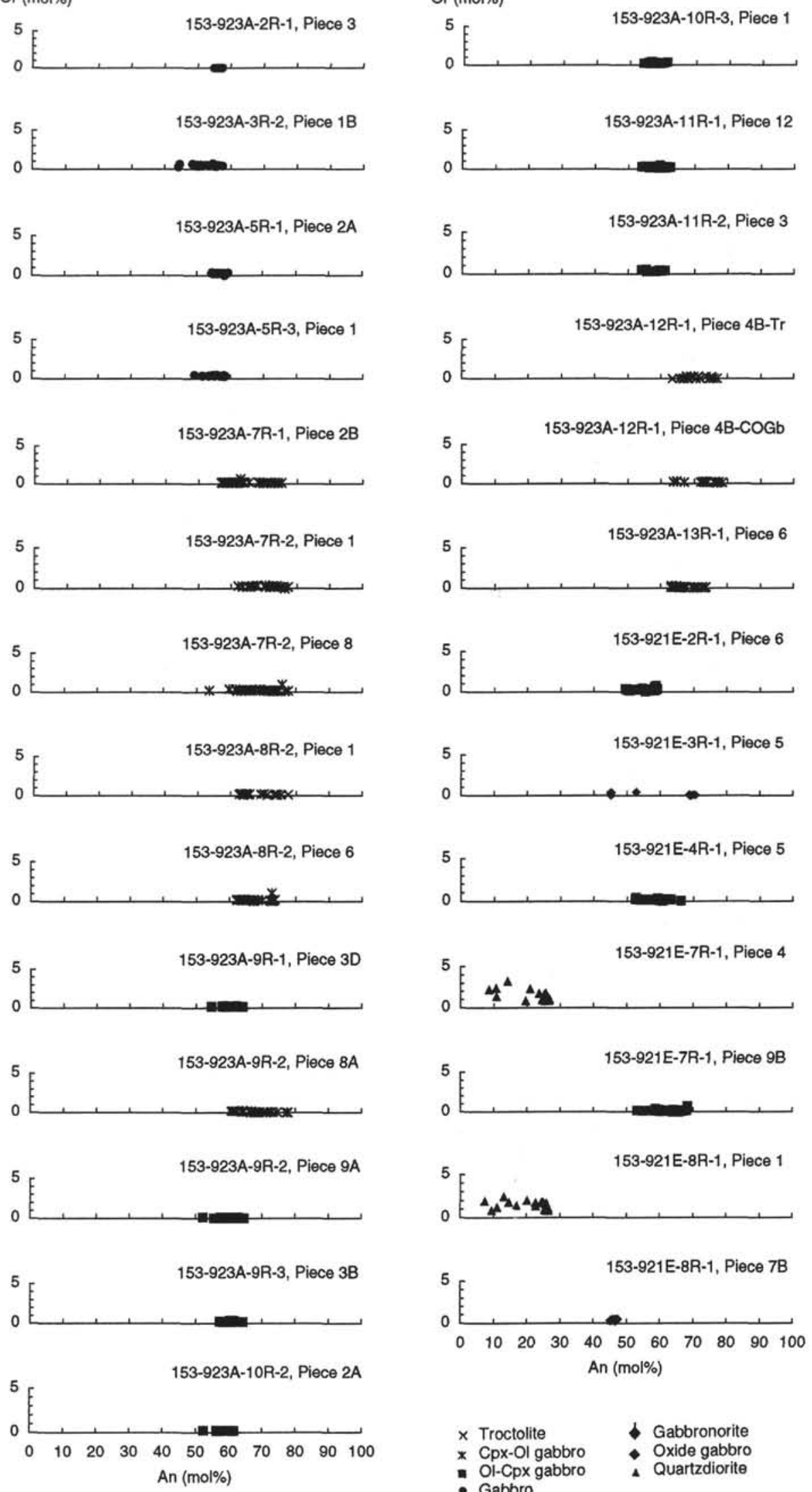

$$
\begin{array}{ll}
\times \text { Troctolite } & \$ \text { Gabbronorite } \\
\text { × Cpx-Ol gabbro } & \$ \text { Oxide gabbro } \\
\text { - Ol-Cpx gabbro } & \star \text { Quartzdiorite } \\
\text { - Gabbro } &
\end{array}
$$



Figure 1. An-Or (mol\%) plot of plagioclase in gabbroic rocks from Holes $921 \mathrm{E}$ and $923 \mathrm{~A} . \mathrm{Cpx}=$ clinopyroxene, $\mathrm{Ol}=$ olivine. 



Fo $(\mathrm{mol} \%)$ in olivine

Figure 2. Fo (mol\%) in olivine in troctolite, clinopyroxene-olivine gabbro, olivine-clinopyroxene gabbro, and gabbro from Holes $921 \mathrm{E}$ and $923 \mathrm{~A}$. Symbols are the same as in Figure 1. 

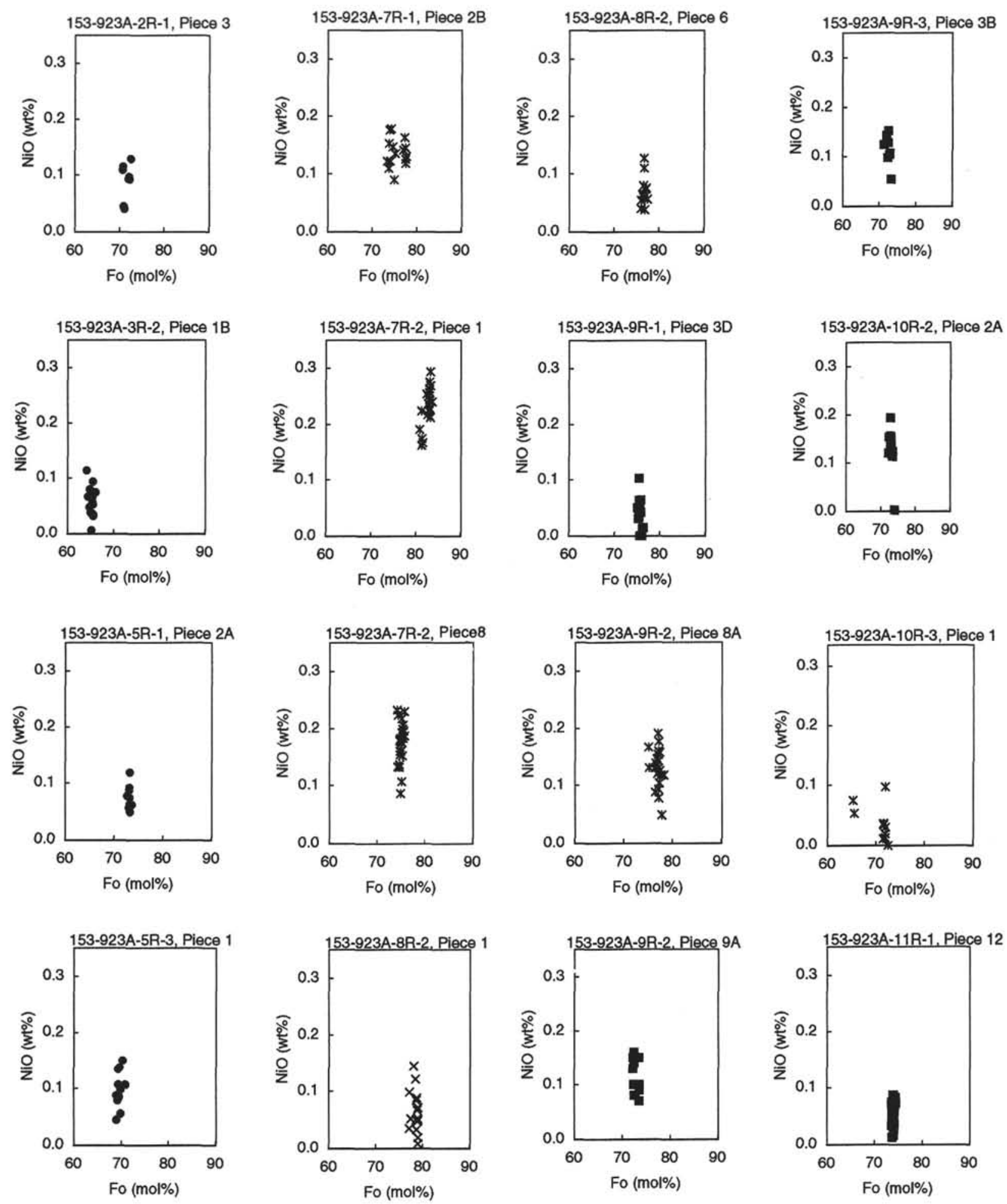

Figure 3. $\mathrm{NiO}(\mathrm{wt} \%)$ in olivine in troctolite, clinopyroxene-olivine gabbro, olivine-clinopyroxene gabbro, and gabbro from Holes $921 \mathrm{E}$ and $923 \mathrm{~A}$. Symbols are the same as in Figure 1. 

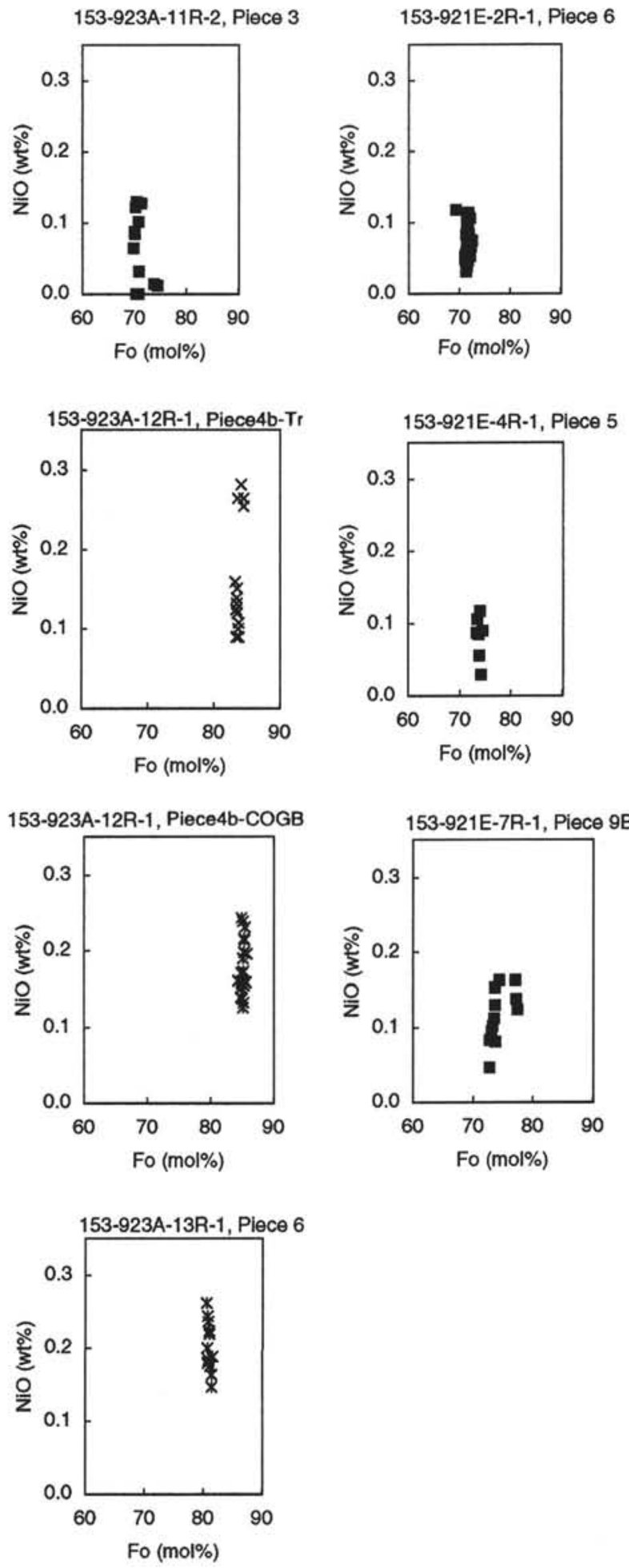

Figure 3 (continued). 
153-923A-2R-1, Piece 3

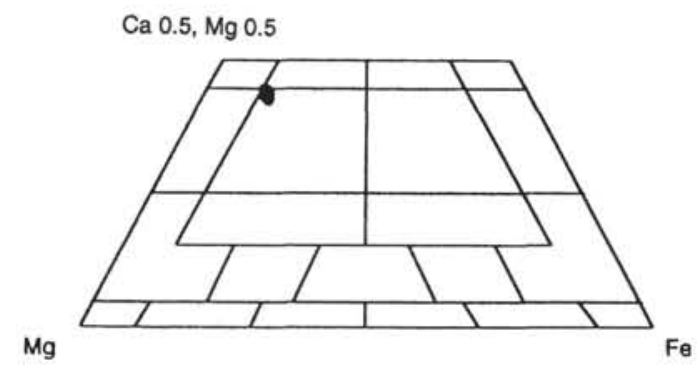

153-923A-3R-2, Piece $1 B$

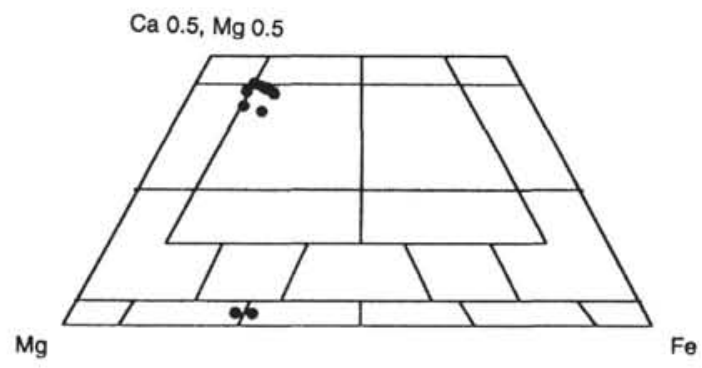

153-923A-5R-1, Piece 2A

Ca $0.5, \mathrm{Mg} 0.5$

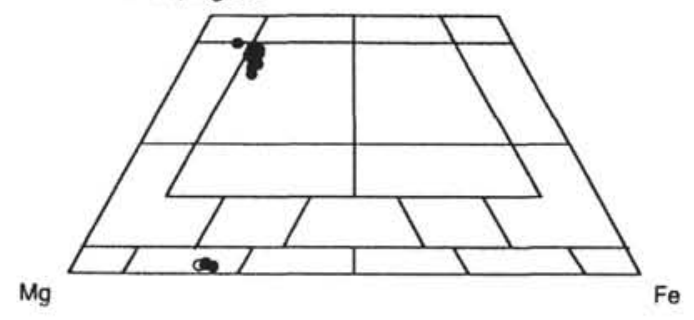

153-923A-5R-3, Piece 1

Ca $0.5, \mathrm{Mg} 0.5$

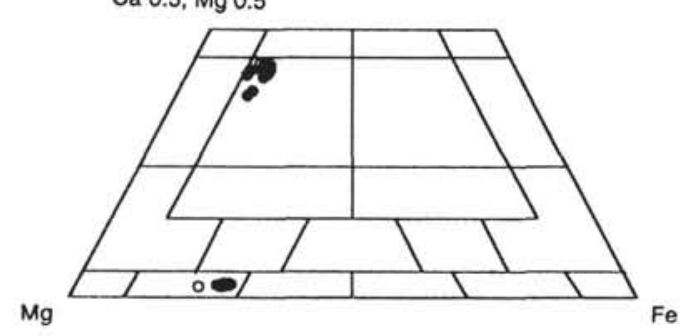

153-923A-7R-1, Piece 2B

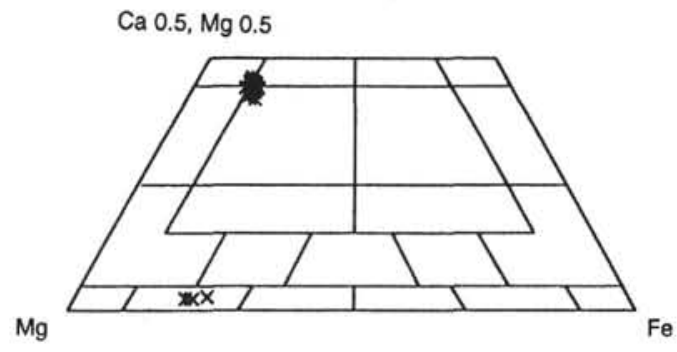

153-923A-7R-2, Piece 1

$\mathrm{Ca} 0.5, \mathrm{Mg} 0.5$

$\mathrm{Mg}$

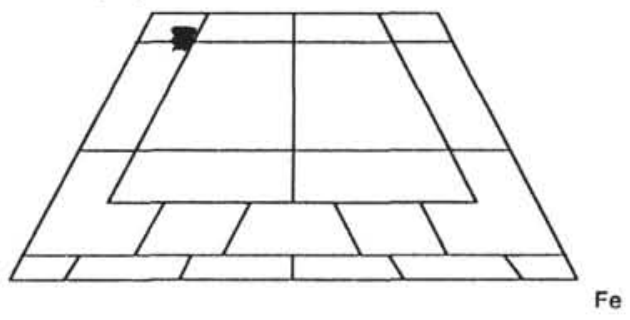

153-923A-7R-2, Piece 8

Ca $0.5, \mathrm{Mg} 0.5$

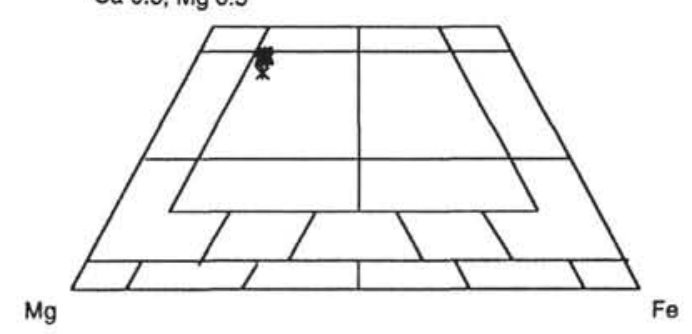

153-923A-8R-2, Piece 1

$\mathrm{Ca} 0.5, \mathrm{Mg} 0.5$

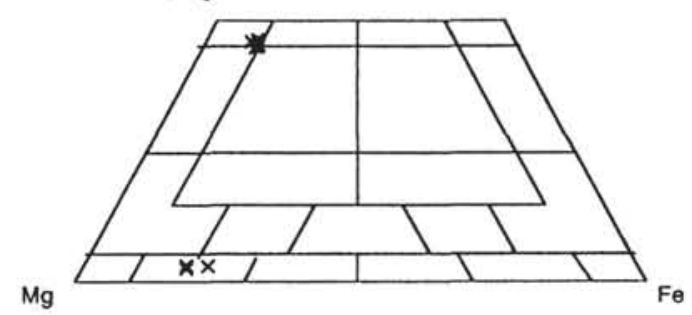

Figure 4. Ca-Mg-Fe (atomic ratio) plot of pyroxenes in gabbroic rocks from Holes $921 \mathrm{E}$ and $923 \mathrm{~A}$. Symbols are the same as in Figure 1 . Open symbols are for exsolution lamellae and host. 
153-923A-8R-2, Piece 6



153-923A-9R-1, Piece 3D

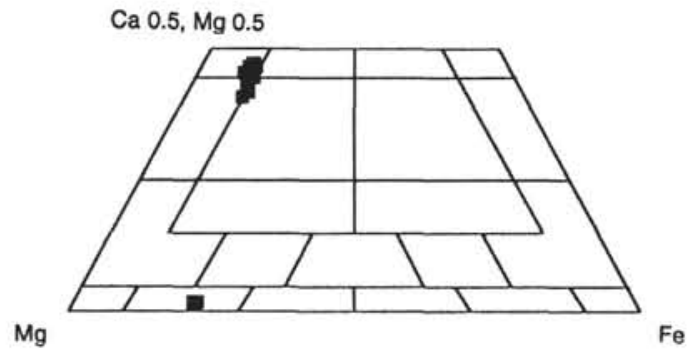

153-923A-9R-2, Piece 9A

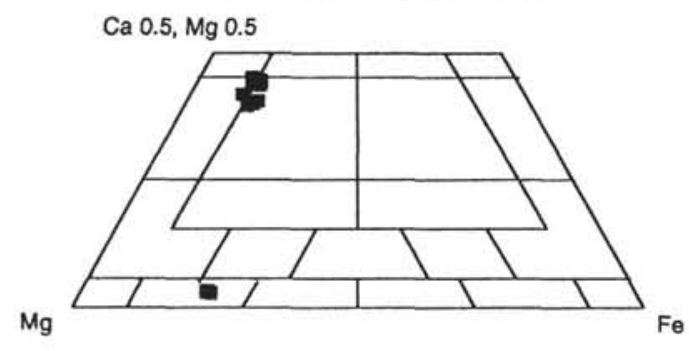

153-923A-9R-2, Piece 8A

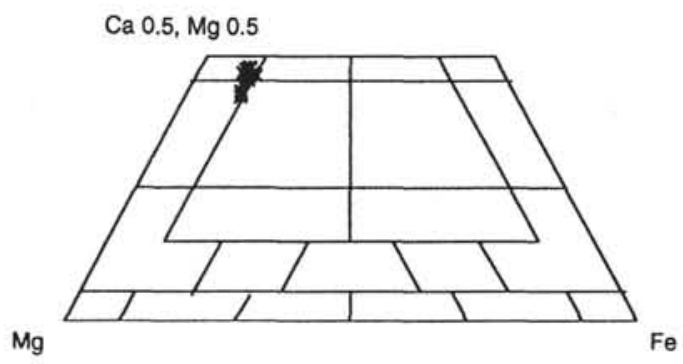

153-923A-9R-3, Piece 3B

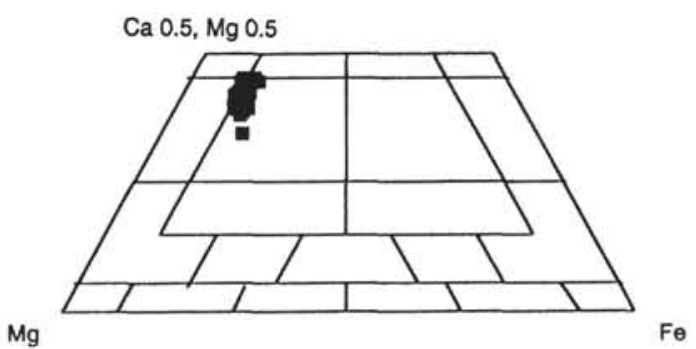

153-923A-10R-2, Piece 2A

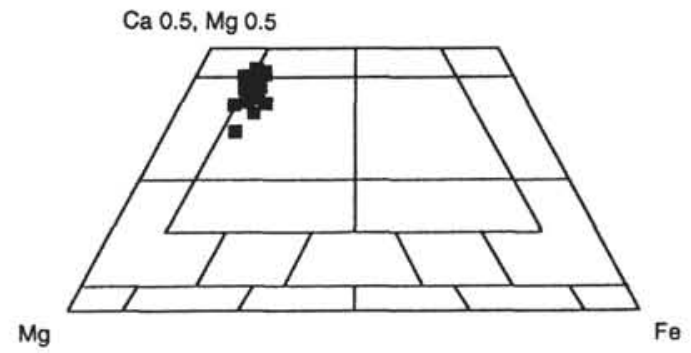

153-923A-10R-3, Piece 1

$\mathrm{Ca} 0.5, \mathrm{Mg} 0.5$

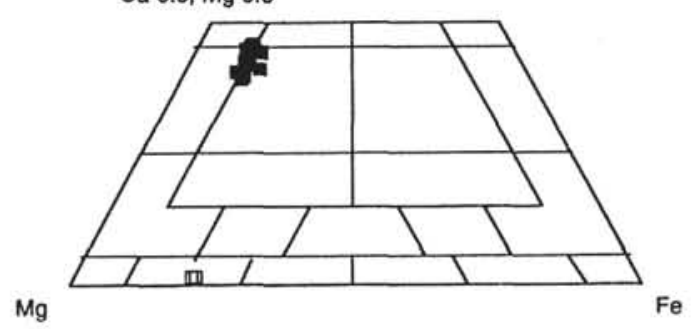

153-923A-11R-1, Piece 12

$\mathrm{Ca} 0.5, \mathrm{Mg} 0.5$

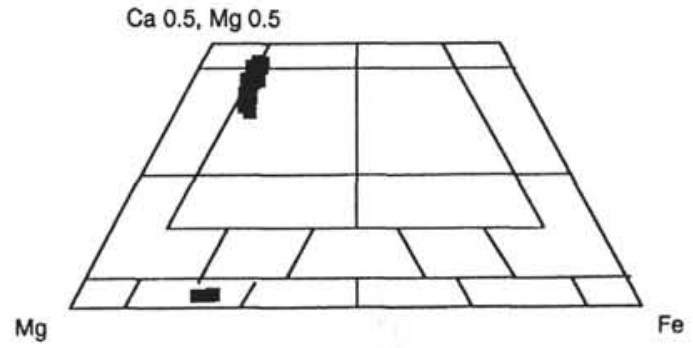

Figure 4 (continued). 
153-923A-11R-2, Piece 3

$\mathrm{Ca} 0.5, \mathrm{Mg} 0.5$

$\mathrm{Mg}$

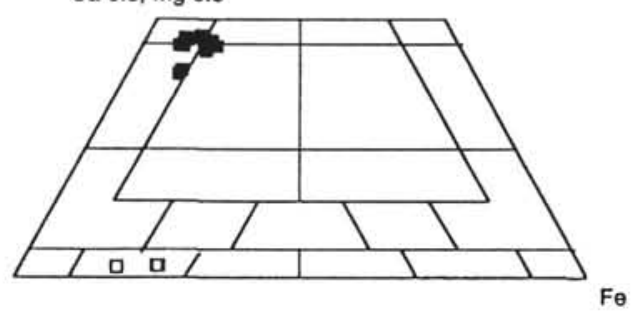

153-923A-12R-1, Piece 4B-Tr

Ca $0.5, \mathrm{Mg} 0.5$

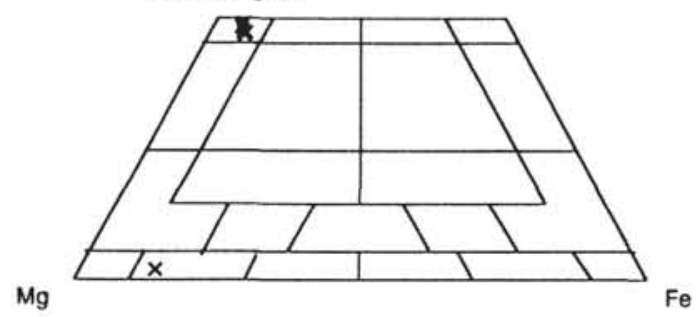

153-923A-12R-1, Piece 4B-COGb

$\mathrm{Ca} 0.5, \mathrm{Mg} 0.5$

$\mathrm{Mg}$

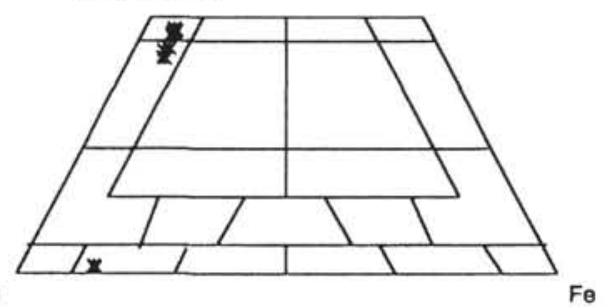

153-923A-13R-1, Piece 6

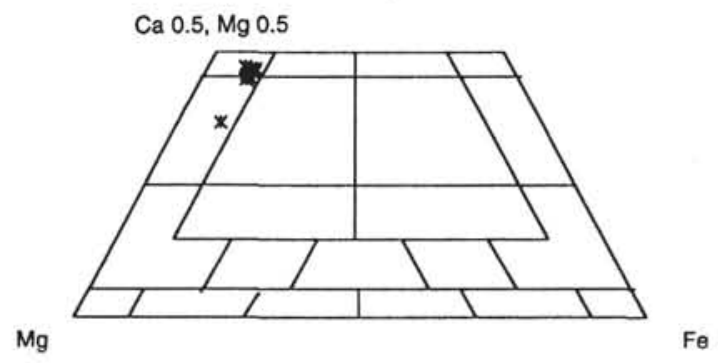

153-921E-2R-1, Piece 6

$\mathrm{Ca} 0.5, \mathrm{Mg} 0.5$

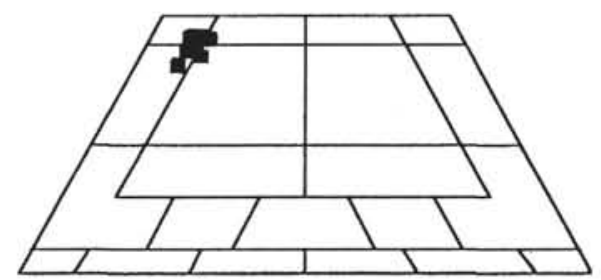

$\mathrm{Mg}$ $\mathrm{Fe}$

153-921E-3R-1, Piece 5

Ca $0.5, \mathrm{Mg} 0.5$

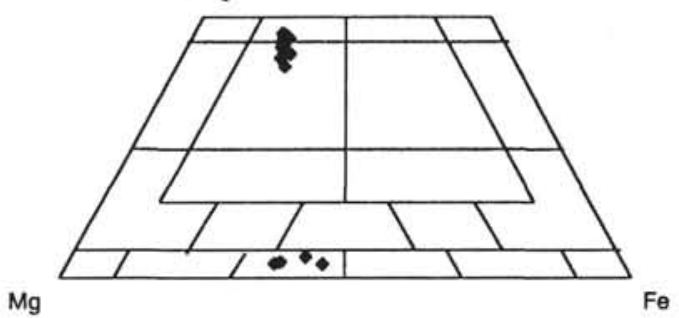

153-921E-4R-1, Piece 5

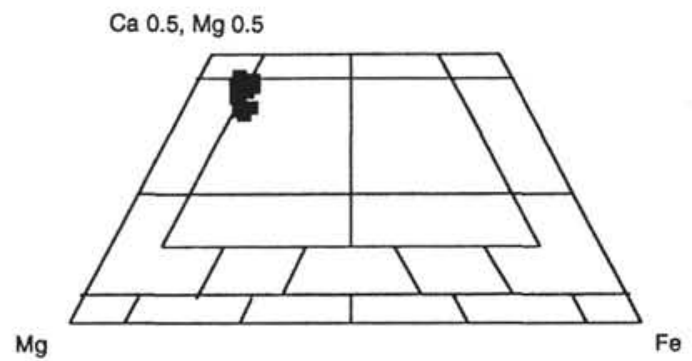

153-921E-7R-1, Piece 4

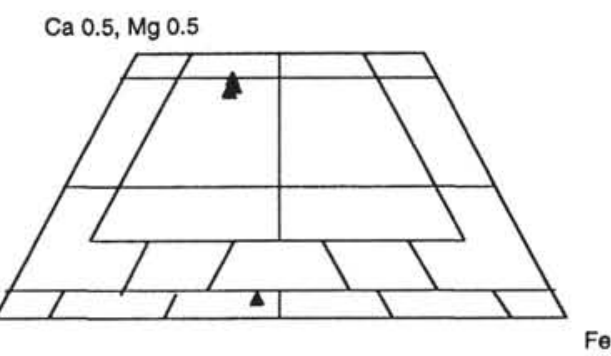

Figure 4 (continued). 
153-921E-7R-1, Piece 9B

Ca $0.5, \mathrm{Mg} 0.5$

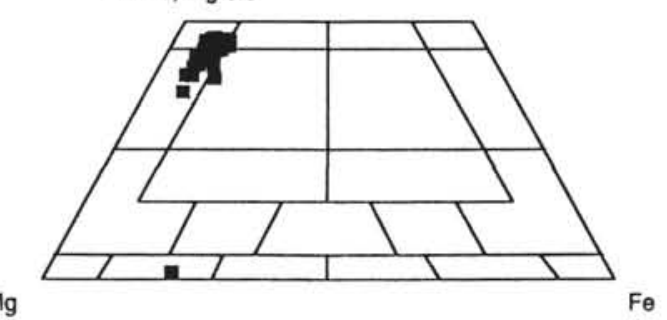

153-921E-8R-1, Piece 1

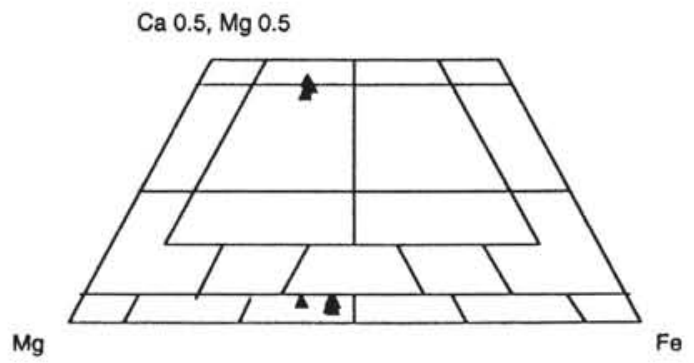

153-921E-8R-1, Piece 7B

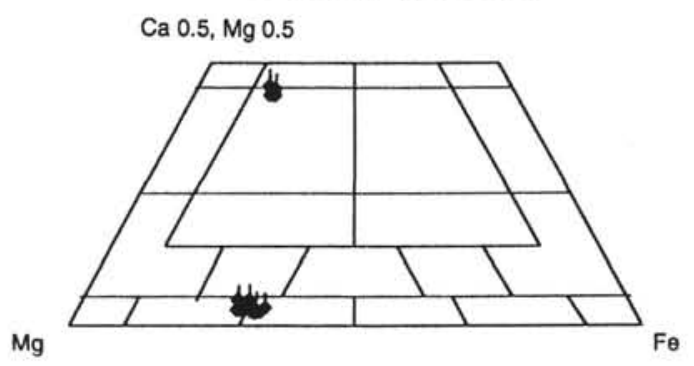

Figure 4 (continued). 

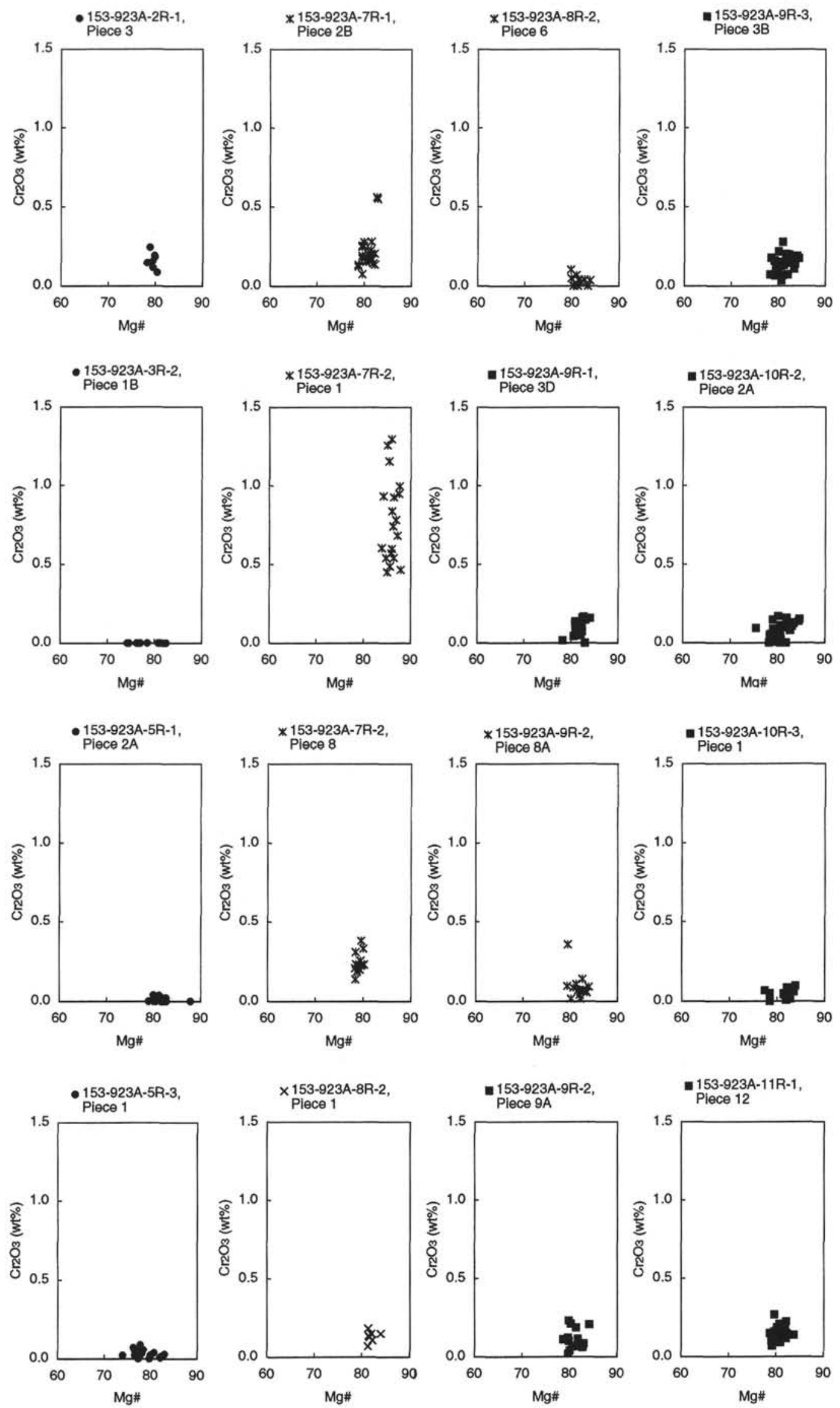

-153-923A-11R-1,

Piece 12

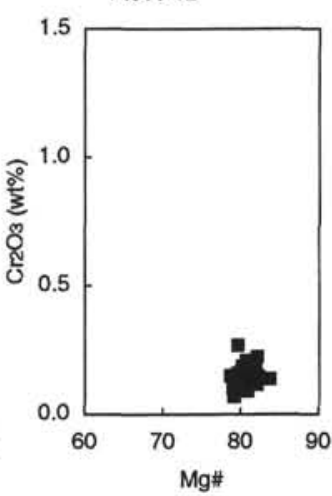

Figure 5. $\mathrm{Cr}_{2} \mathrm{O}_{3}$ (wt\%) in clinopyroxene in gabbroic rocks from Holes $921 \mathrm{E}$ and $923 \mathrm{~A}$. Symbols are the same as in Figure 1. 

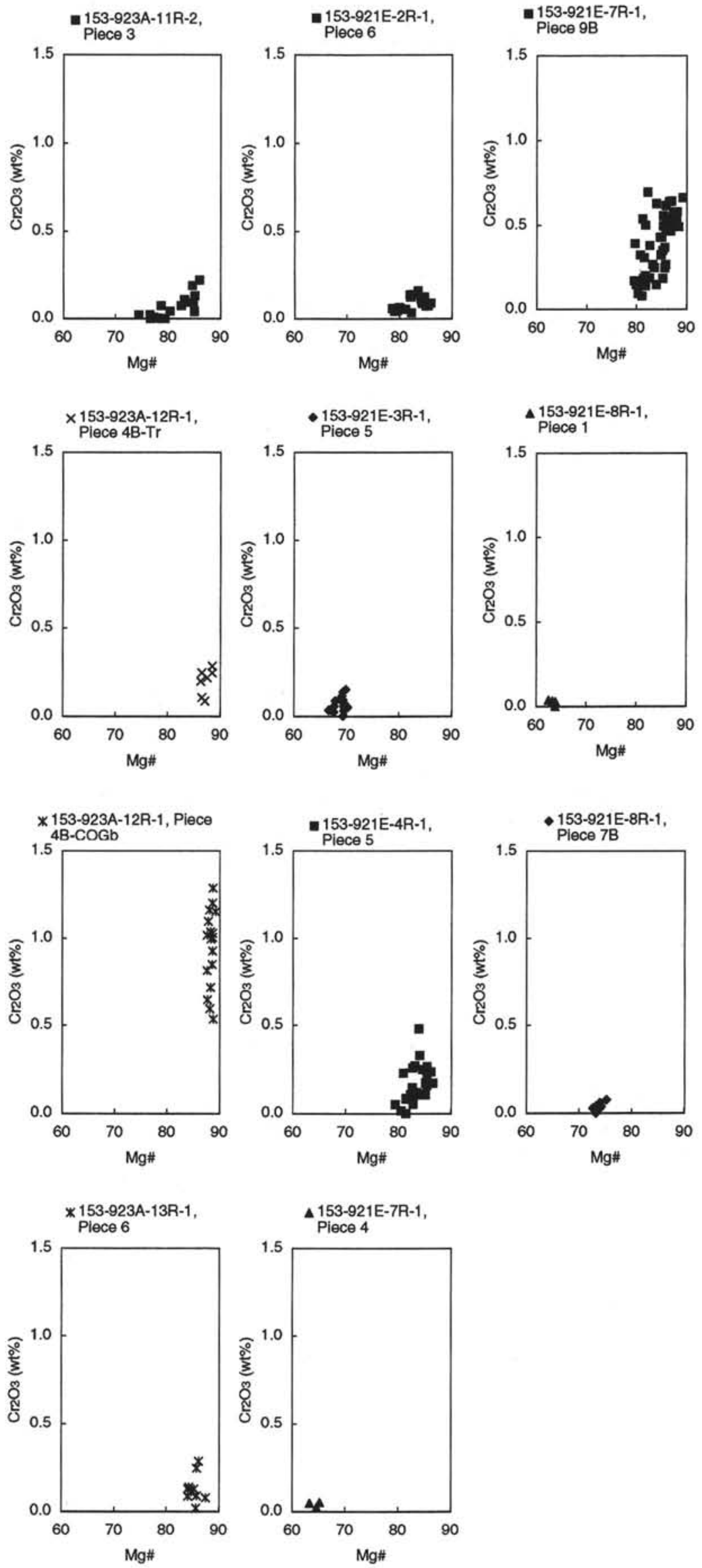

Figure 5 (continued). 
Table 6. Sr- and Nd-isotope compositions for gabbroic rocks from Holes $921 \mathrm{E}$ and 923A.

\begin{tabular}{lllllll}
\hline $\begin{array}{c}\text { Hole, core, } \\
\text { section, piece no. }\end{array}$ & Rock name & Mineral & ${ }^{87} \mathrm{Sr} /{ }^{86} \mathrm{Sr}$ & $2 \sigma$ & ${ }^{143} \mathrm{Nd} /{ }^{144} \mathrm{Nd}$ & $2 \sigma$ \\
\hline 921E-8R-1, Piece 1 & Quartz diorite & $\mathrm{Pl}$ & 0.702452 & 9 & & \\
& & $\mathrm{Px}$ & & & 0.513252 & 29 \\
921E-8R-1, Piece 7B & Gabbronorite & $\mathrm{Px}$ & 0.702445 & 25 & & \\
923A-8R-2, Piece 1 & Troctolite & $\mathrm{Px}$ & 0.702542 & 22 & & \\
923A-9R-2, Piece 8A & Cpx-ol gabbro & $\mathrm{Pl}$ & 0.702316 & 14 & \multirow{2}{*}{0.513243} & 26 \\
& & $\mathrm{Px}$ & & & \\
& & $\mathrm{Bulk}$ & 0.702599 & 16 & & \\
923A-10R-2, Piece 2A & Ol-cpx gabbro & $\mathrm{Px}$ & 0.702403 & 16 & & \\
923A-10R-3, Piece 1A & Ol-cpx gabbro & $\mathrm{Px}$ & 0.702338 & 13 & & \\
\hline
\end{tabular}

Notes: $\mathrm{Cpx}=$ clinopyroxene, $\mathrm{Ol}=$ olivine, $\mathrm{Pl}=$ plagioclase, $\mathrm{Px}=$ pyroxene. 